\title{
Condensin II inactivation in interphase does not affect chromatin folding or gene expression
}

Nezar Abdennur 1,2*, Wibke Schwarzer 3*, Aleksandra Pekowska 4, Indra Alon Shaltiel 5, Wolfgang Huber 4, Christian H Haering 5, Leonid Mirny 2,6, François Spitz 3,7,8

1. Computational and Systems Biology Program, MIT, Cambridge, MA 02139

2. Institute for Medical Engineering and Science, MIT, Cambridge, MA 02139

3. Developmental Biology Unit - European Molecular Biology Laboratory - Heidelberg Germany

4. Genome Biology Unit - European Molecular Biology Laboratory - Heidelberg - Germany

5. Cell Biology and Biophysics Unit - European Molecular Biology Laboratory - Heidelberg - Germany

6. Department of Physics, MIT, Cambridge, MA 02139

7. Department of Developmental and Stem Cell Biology - Institut Pasteur - Paris - France

8. UMR 3738 CNRS

*Joint first authors

\section{Summary}

Condensin complexes have been proposed to play a prominent role in interphase chromatin organization and control of gene expression. Here, we report that the deletion of the central condensin II kleisin subunit Ncaph2 in differentiated mouse hepatocytes does not lead to significant changes in chromosome organization or in gene expression. Both observations challenge current views that implicate condensin in interphase chromosomal domain formation and in enhancer-promoter interactions. Instead, we suggest that the previously reported effects of condensin perturbation may result from their structural role during mitosis, which might indirectly impact the re-establishment of interphase chromosomal architecture after cell division. 


\section{Introduction}

Metazoan chromosomes fold into highly diverse and dynamic conformations constrained by organizational processes that act across genomic length scales (Dekker and Mirny, 2016; Dixon et al., 2016; Gibcus and Dekker, 2013). Chromosomal architectures differ drastically between interphase and mitosis. In interphase, $\mathrm{Hi}-\mathrm{C}$ maps of interphase genomes have revealed distinct cell-type and locus-specific patterns of chromatin contact frequency. These include sequences of alternating intervals that indicate compartmental segregation of active and inactive chromatin (Lieberman-Aiden et al., 2009), topologically associating domains (TADs), which reflect local compaction (Dixon et al., 2012; Nora et al., 2012), and TAD-associated peaks (Rao et al., 2014) and tracks (Fudenberg et al., 2016) of enriched contact frequency between pairs of loci in cis. As cells progress through mitosis, these prominent interphase features disappear as chromosomes get compacted into dense arrays of chromatin loops (Nagano et al., 2017; Naumova et al., 2013). These differences in chromosome organization are tightly related to changes in genomic activities, notably gene regulation in interphase and chromosome segregation during mitosis.

The structural maintenance of chromosomes (SMC) complexes, cohesin and condensin, have been implicated as essential players in the organization of distinct aspects of chromosome organization (Gause et al., 2008; Hirano, 2016; Merkenschlager and Nora, 2016; Rana and Bosco, 2017; van Ruiten and Rowland, 2018). While these two related complexes were originally identified as key determinants of proper segregation of chromosomes during mitosis, a mounting body of evidence has accumulated for their role in the organization of interphase chromosomes (reviewed in (Hirano, 2016; Rana and Bosco, 2017; Yuen and Gerton, 2018)). We and others recently demonstrated that cohesin complexes are essential for the appearance of TADs and their associated patterns (e.g., tracks and peaks) in Hi-C maps (Haarhuis et al., 2017; Rao et al., 2017; Schwarzer et al., 2017; Wutz et al., 2017). The consequences of altered of cohesin dynamics (through inactivation of its loading/unloading factors Nipbl, Mau2 and Wapl) (Haarhuis et al., 2017; Schwarzer et al., 2017) are consistent with the TADs resulting from a loop extrusion mechanism (Fudenberg et al., 2016; Sanborn et al., 2015) (reviewed in (Fudenberg et al., 2018)). Importantly, this cohesin-dependent process is not required for the segregation of active and inactive chromatin into compartments (Rao et al., 2017; Schwarzer et al., 2017; Wutz et al., 2017). Rather, cohesin-mediated loop extrusion interferes with chromatin compartmentalization (Nuebler et al., 2018) showing that TADs and compartments are organized by different principles and mechanisms (Rao et al., 2017; Schwarzer et al., 2017).

While cohesin's involvement in the organization of the interphase genome architecture has by now been clearly established, the function of condensin complexes in the formation of TADs and compartments remains unclear. Most metazoans have two types of condensin complexes, which are composed of the same SMC2-SMC4 dimers that associate with distinct non-SMC subunits (Ono et al., 2003). Condensin I and II serve different functions in the compaction of mitotic chromosomes (Green et al., 2012; Ono et al., 2003). During interphase, only condensin II is reportedly present in nuclei, whereas condensin I is sequestered in the cytoplasm (Gerlich et al., 2006; Hirota, 2004; Ono et al., 2004), although recent data indicate 
that a small fraction of condensin I may be also be in the nucleus during interphase (Dowen et al., 2013; Li et al., 2015; Zhang et al., 2016). In C. elegans, a distinct condensin complex (condensin $\mathrm{I}^{\mathrm{DC}}$ ) has been described and shown to be essential for the organization of TAD-like domains in hermaphrodite $X$ chromosomes (Crane et al., 2015). This overall structure leads to a chromosome-wide reduction in gene expression that contributes to balancing expression levels between XX hermaphrodite and XO male animals (Crane et al., 2015; Jans et al., 2009).

In other species, experimental evidence suggested that condensin II contributes to interphase chromosomal organization and gene expression. In Drosophila, mutations in the condensin II kleisin subunit Ncaph2 reduce the axial compaction of chromosomes, which is thought to be required for favoring intra- over inter-chromosomal interactions and the establishment of chromosome territories (Bauer et al., 2012). ChIP-seq studies reported colocalization of condensin II with other architectural and insulator proteins at the borders of Drosophila domains, suggesting that it could contribute to domain boundary formation or strength (Van Bortle et al., 2014). This pattern, which is mediated by interaction with TFIIIC, appears to be conserved in mice and humans (Yuen et al., 2017). Supporting the notion that the binding of condensin II to chromosomes contributes to gene regulation, knockdown of NCAPH2 was proposed to lead to down-regulation of genes close to TAD boundaries (Yuen et al., 2017). Several other reports provided circumstantial evidence implicating condensin I and II in the control of gene expression (Kranz et al., 2013; Schuster et al., 2013; Woodward et al., 2016), although the underlying mechanisms might have been indirect in most of these cases. Noteworthy, recent studies suggested the recruitment of condensin complexes to generegulatory elements, notably super-enhancers (Dowen et al., 2013; Li et al., 2015), where they were proposed to contribute to enhancer activation and looping interaction with promoters (Li et al., 2015) and sustained expression of cell-identity genes (Dowen et al., 2013). However, the experimental setup used in the later studies (either dividing embryonic stem (ES) cells or interference performed as cells are stripped from serum but not yet fully blocked in G0/G1) does not exclude the possibility that the observed changes are indirect consequences of mitotic defects that resulted in aberrant chromosome organization upon exit from mitosis.

To address the uncertainty regarding the role of the condensin II complex in interphase genome organization and gene expression, we deleted the central condensin II kleisin subunit Ncaph2 in post-mitotic adult mouse hepatocytes. Remarkably, this deletion did not result in any measureable alterations in chromatin organization, as compared to wild-type and Nipbl deletions as negative and positive controls, respectively. TADs and compartments appear unaffected. The expression level of only a handful of genes show significant changes and most of those genes are functionally related to mitosis. Our data suggest that, in contrast to cohesin, condensin II is dispensable for the organization of chromosomal domains in non-dividing cells and that its direct contribution in the control of interphase gene expression is negligible.

\section{Results and Discussion}

To determine the functions of condensin II complexes in interphase chromatin folding and gene expression, we deleted critical exons of the gene encoding the Ncaph2 kleisin subunits (figure 1A), which is essential for the activity of the condensin II complex during mitosis. Homozygous deletion of Ncaph2 is lethal in mice and leads to rapid death of proliferating cells 
(Nishide and Hirano, 2014). To avoid confounding effects, we used a tissue-specific conditional strategy to induce acute deletion of the gene in non-dividing cells. We achieved this by

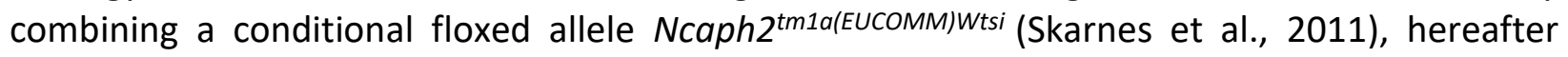
referred to as Ncaph2 $2^{\text {flox }}$, with a liver-specific tamoxifen-inducible CRE driver Tg(Ttrcre/Esr1*)1Vco (Tannour-Louet et al., 2002). We previously used the same strategy to successfully delete the cohesin loading factor Nipbl (Schwarzer et al., 2017) and thereby demonstrated its requirement for TAD formation. We followed the same procedure for Ncaph2. Ten days after tamoxifen injections, we collected hepatocytes from Ncaph2 flox/flox ; $\mathrm{Tg}\left(\text { Ttr-cre/Esr1 }{ }^{*}\right)^{1 V c o}$ and control animals (figure 1B). Ncaph2 deletion was extremely efficient as judged by mRNA and protein level quantification (figure 1C-F).

To examine the effects of condensin II knockout on genome organization, we performed tethered chromatin conformation capture (Kalhor et al., 2012), referred below as Hi-C. For each condition, we produced two different biological replicates (table S1). The data from replicates showed high degree of correlation (figure 1E,F, S2A, S3A) and we therefore pooled them in subsequent analyses. We compared key features of $\mathrm{Hi}-\mathrm{C}$ maps, including the global scaling of contact frequency $P(s)$, compartmentalization, contact insulation, contact frequency peaks, and aggregate maps of genomic landmarks associated with chromatin architecture. Unlike the dramatic effects that result from the loss of chromatin-associated cohesin in $\Delta \mathrm{Nipbl}$ (Schwarzer et al., 2017), we observed no substantial changes upon inactivation of condensin II. Examination of $\mathrm{Hi}-\mathrm{C}$ maps did not reveal alterations of TAD or compartmentalization patterns (figure 1G).

To analyze the contact maps of condensin II-inactivated cells in more detail, we calculated the curves of the contact probability $P(s)$ as a function of genomic separation $s$. The proximal shallow region of the $P(s)$ curve $(s \approx 100-300 \mathrm{~kb}$ ), referred to as the shoulder (Fudenberg et al., 2018), reflects local compaction. This region is sensitive to the loss of cohesin, as seen across several studies, consistent with the loss of compaction by loop extruding cohesins. By contrast, the shoulder of $P(s)$ cells did not differ between hepatocytes from $\Delta$ Ncaph2, WT or tamoxifeninjected (TAM) control animals (figure 2A, S1A). We also failed to identify any apparent difference for $P(s)$ restricted to locus pairs within and between wildtype domain intervals (mostly TADs) and within and between A/B compartmental intervals (figure S1B,C).

The most striking effect of cohesin removal is the ubiquitous loss of the features that collectively make up mammalian TADs (local enrichment/insulation, tracks, corner peaks, (Fudenberg et al., 2018)). In contrast with $\Delta$ Nipbl (Schwarzer et al., 2017), average maps of TADs in $\triangle$ Ncaph2 do not differ from those of controls (figure 2B). Similarly, no effect is observed in average $\mathrm{Hi}-\mathrm{C}$ peak maps, where $\Delta \mathrm{Nipbl}$ resulted in a fourfold reduction in contact frequency, nor in the asymmetric average contact footprint of CTCF sites which disappears in $\Delta$ Nipbl (figure 2C,D).

Next, we assessed changes in A/B compartmentalization, which reflects segregation of active and inactive chromatin, and is manifested as genome-wide checkerboard patterns on $\mathrm{Hi}$ C maps (Imakaev et al., 2012; Lieberman-Aiden et al., 2009) (figure 2E,F). The strength of compartmentalization was increased in studies of acute cohesin degradation (Rao et al., 2017; Wutz et al., 2017), and in the case of persistent cohesin removal via Nipbl deletion in postmitotic liver cells, we additionally observed the emergence of a finer compartmental segmentation in large gene-dense regions, quantifiable as a steeper decay of the compartment 
signal's autocorrelation function (Schwarzer et al., 2017). In sharp contrast, Ncaph2 deletion did not result in any of these effects (figure $\mathbf{2 G}, \mathbf{H}$ ). Indeed, the $\Delta$ Ncaph2 and control eigenvectors were strongly correlated (figure $\mathbf{2 E}, \mathbf{F}$ ) and there was no change in the autocorrelation function of the eigenvector compared with controls (figure $\mathbf{2 G}$ ). Taken together these results show that condensin appears to play no measurable role in the formation of TADs and associated features, nor in compartmental segregation of active and inactive chromatin in interphase.

Since the loss of chromatin-associated cohesin resulted in genome-wide changes in the diamond insulation score profile, we also examined changes in contact insulation (figure 2I,J) in Ncaph2 mutants. The minima of the insulation score profile correspond to loci for which the contact frequency between neighboring loci on opposite sides is relatively depleted - typically the boundaries of TADs and of compartmental intervals (Crane et al., 2015). The loss of TADs in $\triangle \mathrm{Nipbl}$ cells led to dramatic changes in the insulation profile, as it can be observed in the scatter plot of genome-wide insulation scores against WT. However, the $\Delta$ Ncaph2 insulation profile correlates as strongly with WT as does that of the TAM control condition. The lack of effect of Ncaph2 deletion was further highlighted in the depth of the insulation score "valley" averaged around insulating loci (boundaries) identified in WT, which was preserved in TAM and $\triangle$ NCaph2 but raised in $\Delta \mathrm{Nipbl}$ (figure $\mathbf{2 K}$ ).

We further tested whether Ncaph2 deletion may have caused more fine-grained specific changes in local insulation. To detect differentially insulating (DI) loci we used an approach similar to that employed to detect differentially expressed genes (see Methods). For comparison, we applied the same analysis on $\Delta \mathrm{Nipbl}$ cells (having an established effect) and the TAM control cells versus WT. Using the same stringency cutoff, we detected 752 insulating loci as having a significantly different insulation score in $\Delta$ Nipbl cells vs WT, while only 39 of loci cells were differentially insulating in $\Delta$ Ncaph2 against WT (figure 2L). We found also 39 differentially insulating loci when we compared TAM against WT, and a similar number was obtained when we apply the same procedure on control comparisons of insulation changes between replicates within the same condition (figure S3). This implies that the detectable DI loci upon condensin loss are largely false positives and not significant. We conclude that local chromosome organization remains unchanged upon loss of condensin II in interphase.

To test for more subtle or specific effects, we focused on specific regions that have been previously associated with condensin II function. Condensin II has been implicated in certain genomic regions including (1) histone gene clusters, (2) super-enhancers, in mouse ES cells, (3) TFIIIC and tRNA genes, in Schizosaccharomyces pombe and mammals. For each of these features we examine changes in interaction frequencies between the loci. We first examine the interaction between the two histone clusters present on chromosome 13, which was reported to depend on condensin II (Yuen et al., 2017). We indeed detect particularly strong interaction between the two clusters, but did not note differences between $\Delta \mathrm{Ncaph} 2$ and controls (figure 3A). We then examined interactions between super enhancers (Pott and Lieb, 2014), for which we considered a curated list for mouse liver (Khan and Zhang, 2016) (figure S4) or identified such regions de novo by locally stitching mouse liver H3K27ac sites as in (Hnisz et al., 2013) (figure 3B). We saw little enrichment of enhancer-enhancer interactions above the level observed for regions of the same expression level and hence explained by compartmentalization of euchromatin. In all cases, there was no significant change in enrichment between $\Delta \mathrm{Ncaph} 2$ and controls, while a mild increase in pairwise contact frequency 
could be detected in $\Delta \mathrm{Nipbl}$, consistent with increased compartmental segregation upon cohesin loss (Rao et al., 2017; Schwarzer et al., 2017). We then examined the role of condensin in organizing RNAP-III-associated regions. We produce average contact profiles centered at paired POLR3D (RPC4) (figure 3C) and POLR3A (RPC1) (figure S5) binding sites identified by ChIP-seq in mouse liver (Canella et al., 2012) as well as at the locations of TFIIIC ChIP-seq sites identified in mouse ES cells (Yuen et al., 2017) (figure S5). Again, we saw no significant change in enrichment between $\Delta \mathrm{Ncaph} 2$ and controls. Finally, we examined the average Hi-C map centered at the coordinates of pairs of convergently oriented CTCF sites: contact enrichment was lost in $\Delta \mathrm{Nipbl}$ but preserved in $\Delta$ Ncaph2 (figure $3 \mathrm{D}$ ), just as it was for $\mathrm{Hi}-\mathrm{C}$ peak coordinates.

In brief, at the level of resolution and depth offered by our Hi-C data (10-20 kb, 52-75 million valid pairs in cis at $10-\mathrm{kb}$ separation or greater, on par with that of other $\mathrm{Hi}-\mathrm{C}$ of primary tissues (Schmitt et al., 2016)), we did not identify significant differences between the structural partitioning of normal and condensin II-deficient chromosomes. Even though we cannot formally exclude a role in features that are difficult to assess with $\mathrm{Hi}-\mathrm{C}$ (e.g., spatial positioning within the nucleus (Falk et al., 2018) or nucleus size, (Fazzio and Panning, 2010; George et al., 2014)) or in highly repetitive regions (e.g., centromeres, pericentric regions), we conclude that condensin is involved neither generally nor specifically in the maintenance of interactions that impinge upon most chromosomal loci.

We next investigated if the absence of Ncaph2 led to gene expression changes (figure 4). For this, we compared the transcriptome of control TAM and $\Delta$ Ncaph2 liver hepatocytes (4 biological replicates per condition). A statistical analysis with DESeq2 (Love et al., 2014) showed that only a very small number of genes were differentially expressed between TAM and Ncaph2-deficient hepatocytes (table 1). Gene ontology analysis of these 64 genes showed strong enrichment for genes associated with cell-cycle and cytokinesis (table 2). About 20 genes that had been reported to show expression changes during cell cycle (predominantly upregulated in $\mathrm{G} 2 / \mathrm{M}$ ) were up-regulated in $\Delta$ Ncaph2 cells. This coherent shift most likely represents a cell-cycle defect in the few hepatocytes that underwent through mitosis during the course of the experiment in order to maintain liver homeostasis, as knockdown of condensin II has been shown to lead to extended prometaphase (Green et al., 2012). Most of the other mis-regulated genes have been shown to exhibit a circadian pattern of expression (Fang et al., 2014; Wang et al., 2018). The predominance of circadian genes (defined as gene showing an expression profile varying with at least a 2-fold amplitude during a 24-h period) is highly significant (two-tailed Chi-square test with Yates' correction, $P<0.0001$ ). However, the correlation between down/up-regulation and normal peak period of the genes is consistent with a shift of a few hours between the two groups of animals and therefore is more likely to correspond to differences in time of sample collection, rather than being a real drift in circadian rhythm (figure S6). Thus, altogether, it appears that the deletion of Ncaph2 and the absence of condensin II activity had an extremely limited effect on gene expression in adult hepatocytes.

The absence of noticeable effects of a deletion of Ncaph2 in non-dividing adult hepatocytes, as shown here, indicates that condensin II has no major function in maintaining interphase chromosome organization and gene regulation. One can argue that condensin I complexes - assuming that they are present in sufficient number in interphase nuclei - may compensate for the absence of condensin II in interphase. While we cannot formally exclude 
this hypothesis, our conclusion on the dispensability of condensin for interphase chromosomal organization is strengthened by a recent study in chicken DT40 cells, which reported major changes in mitotic chromosomes, but no significant differences the interphase chromosomes after acute degradation of either SMC2 or NCAPH2 in G2 phase (Gibcus et al., 2018) (reanalyzed in figure 5).

Our findings that condensin II is largely dispensable for interphase gene expression and does not contribute noticeably to the maintenance of the different folding features characteristic of interphase chromatin contrast with several previous reports which argued that condensin does play an important role in the regulation of interphase mammalian chromosomal architecture (Dowen et al., 2013; Li et al., 2015; Yuen et al., 2017). However, the experiments in those studies were performed under very different conditions. Adult hepatocytes -- the system used here -- are slow-dividing and long-lived, producing perhaps two cell divisions over a period of 300 days (Magami et al., 2002). By contrast, the previous analyses of condensin function in mammalian cells were carried out using rapidly dividing cultured cell lines. Two of these studies ((Dowen et al., 2013; Yuen et al., 2017)) used mouse embryonic stem cells grown under standard culture conditions, which typically have a 10-12 hour-long cell division-cycle (Coronado et al., 2013), and the effects of knockdown experiments were assessed 3-5 days after knockdown. In the third study (Li et al., 2015), NCAPH2 knock-down was initiated as MCF7 cells were progressively arrested from proliferation by serum deprivation, and its consequences were analyzed four days later. Even though this led to a final situation with 8095\% of the cell population in G0/G1 (Li et al., 2015), with a cell cycle length of approximately 20h (Cos et al., 1996) the majority of the originally asynchronous cell population would have completed one to three cell cycles before arresting at the mitogenic restriction point. Thus, these experimental designs leave open the possibility that the observed changes may result from defective mitotic condensation and segregation.

Condensin plays an essential role in mitotic chromosome assembly and segregation. Chromosomes are not correctly segregated if they exit mitosis as intermingled and potentially damaged structures, which can lead to global chromosomal instability and DNA damage responses (Ganem and Pellman, 2012), thus impacting gene expression and chromosome organization in the subsequent interphase. Consistent with this, recent studies in yeast also suggest that the regulatory changes associated with condensin deletion stem from indirect consequences of chromosomal instability rather than from a direct effect of condensin on transcription (Hocquet et al., 2018). Furthermore, in vertebrate cells, condensin complexes have been shown to occupy previously active promoters during mitosis, and this was proposed to "bookmark" them to allow efficient re-expression of the corresponding genes in G1 (Kim et al., 2013). Such ripple effects of mitotic perturbations may account for the previously observed consequences of condensin depletion in interphase cells, while they cannot impact our non- (or very rarely) dividing hepatocytes.

In addition to consequences of chromosome mis-segregation, the axial compaction of chromosomes initiated by condensin II and the lateral compaction subsequently reinforced by condensin I define a very specific organization, wherein each chromosome emerges at the end of mitosis as an individualized entity (Gibcus et al., 2018). Such an initial configuration may be part of the necessary steps for proper establishment of intra- and inter-chromosomal modes of organization in interphase. Accordingly, chromosomal territories (Bauer et al., 2012; Rosin et 
al., 2018), and at a smaller scale cis-interactions between highly active gene clusters (Yuen et al., 2017) and super-enhancers (Dowen et al., 2013), which have been shown to dependent on condensin activity, may be compromised if chromosomes exit mitosis as partially decondensed, intermingled structures as seen in condensin II-depletion mitosis (Gibcus et al., 2018; Hirano, 2016). Our present data argue that this dynamic organization, once established after cell division, does not require condensin II for its maintenance.

Altogether, our data provide evidence that contrarily to what has been previously suggested, condensin II complexes are dispensable to the maintenance of the different organizational modes adopted by interphase chromosomes in vertebrates. Instead, most of the effects of condensin depletion previously reported in "interphase" mammalian cells regarding chromosomal 3D interactions and gene regulation may stem from the indirect consequences of defective mitosis. 


\section{Acknowledgments}

We thank the EMBL Laboratory Animal Resources Facility for animal husbandry and the EMBL Genomics Core Facility for genomic library sequencing. We thank EUCOMM and the Wellcome Trust Sanger Institute for generously providing the Ncaph2 conditional mutant line. We thank John Marioni and Nuno Fonseca (EMBL/EBI) for advice with RNA-Seq data processing. W.S. and A.P were supported by an EMBL Interdisciplinary Postdoc (EIPOD) fellowship, under the Marie Sklodowska Curie Actions COFUND program. This work was partially supported by NIH (GM114190) and NSF,and by the Center for 3D Structure and Physics of the Genome of NIH 4DN Consortium (DK107980) to LM, and NIH Common Fund Program, grant U01CA200147, as a Transformative Collaborative Project Award to LM and FS. Work in the Spitz lab was supported by the European Molecular Biology Laboratory, the Institut Pasteur and the Deutsche Forschunggemeinschaft (DFG SP 1331/3-1). Funding from the European Commission's seventh framework program through the collaborative research project RADIANT (Grant \# 305626, to W.H.) contributed partially to this work.

\section{Author Contributions.}

W.S., C.H.H. and F.S. conceived the study and designed the experiments. W.S. generated experimental data with the help of A.P and I.A.S. W.H. provided resources and advice in preliminary data analysis. N.A. performed all computational analysis. L.M. provided advice and guidance on data analysis. N.A., W.S, C.H.H. and L.M. and F.S. analyzed the results and wrote the paper with input from the other authors. 
bioRxiv preprint doi: https://doi.org/10.1101/437459; this version posted October 7, 2018. The copyright holder for this preprint (which was not certified by peer review) is the author/funder, who has granted bioRxiv a license to display the preprint in perpetuity. It is made available under aCC-BY 4.0 International license.

\section{Figure Legends}

\section{Figure 1: Experimental Strategy}

A

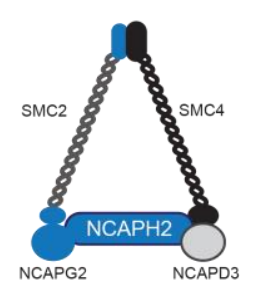

D

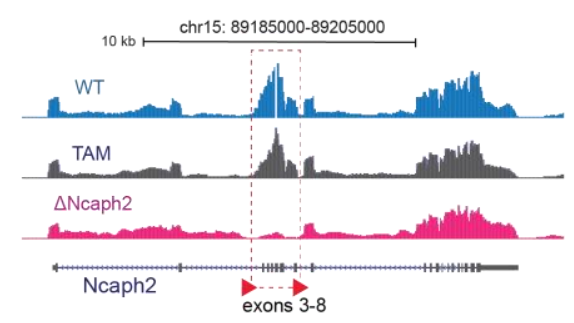

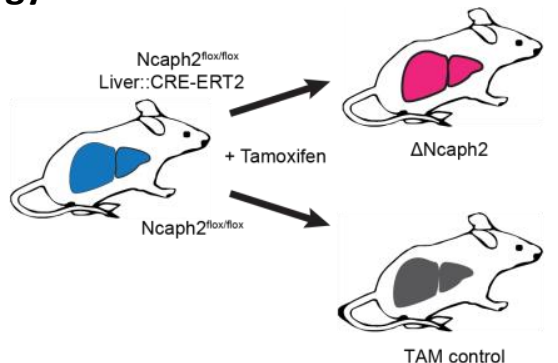

$\mathrm{E}$

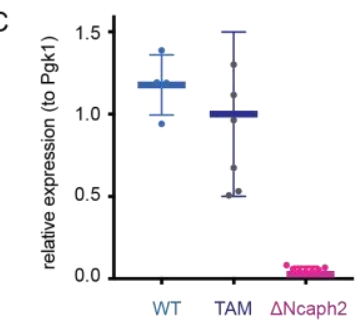

F

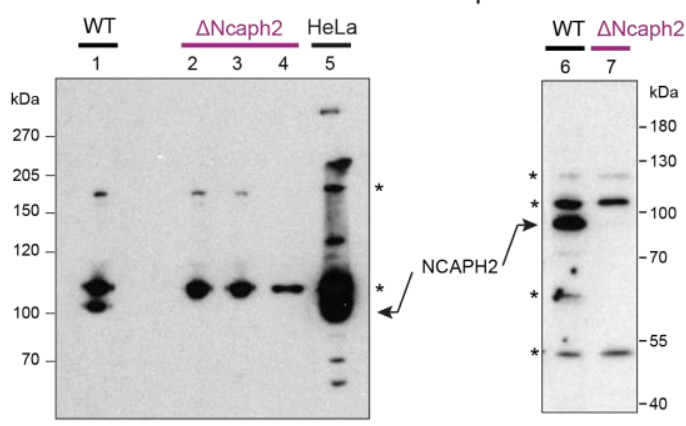

G
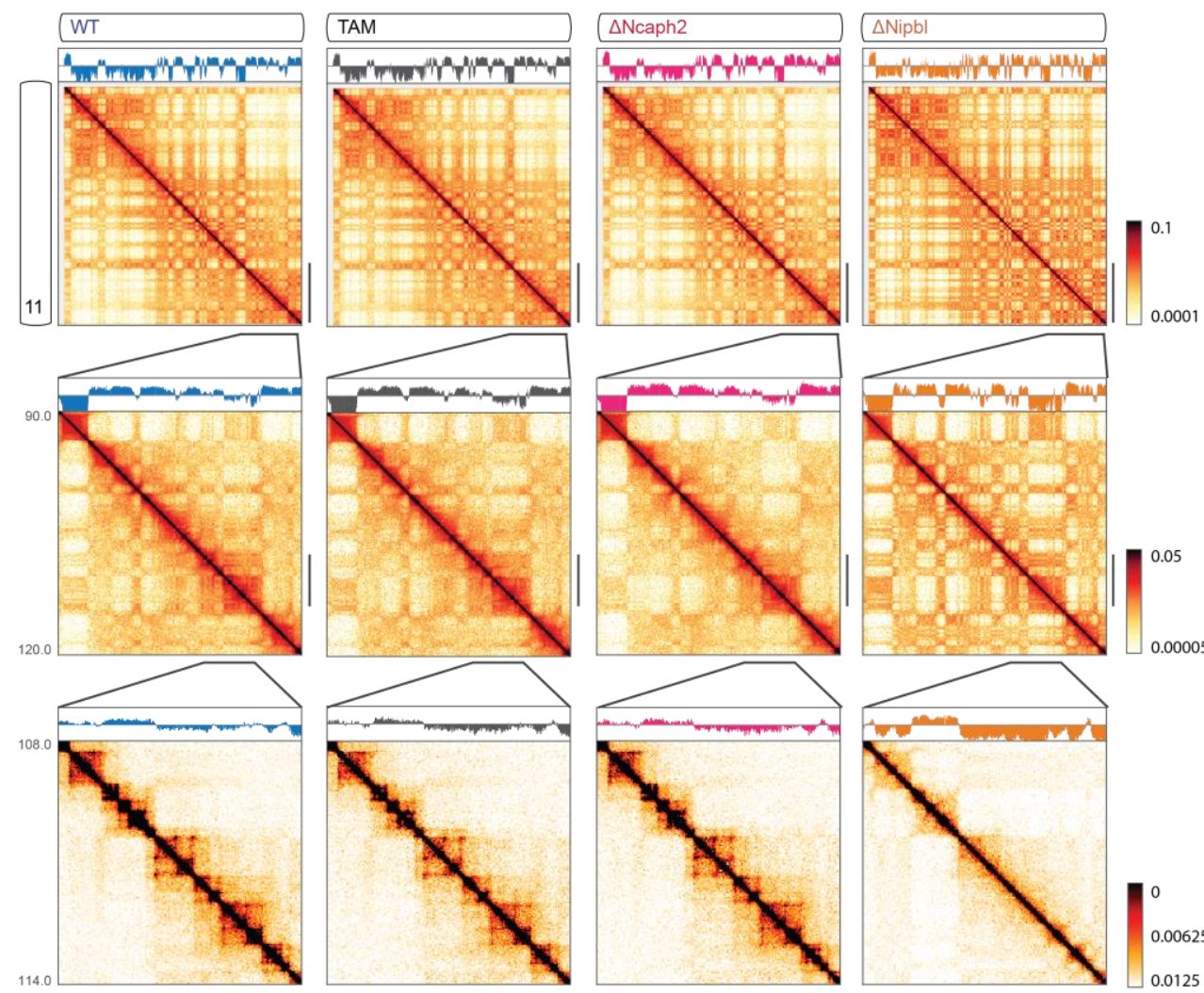

0.00005

0.00625

0.0125 
(A) Schematic representation of the condensin II complex and its subunits. (B) Different groups of mice analyzed. Animals with $\Delta \mathrm{Ncaph} 2$ hepatocytes were produced by intraperitoneal injection of tamoxifen in Ncaph2 $2^{f l o x} / f l o x, T g(T t r-c r e / E s r 1 *)^{1 V c o}$ adult mice. Two different controls were used, either untreated (U) animals (genotype: Ncaph2flox/flox, $T g\left(T t r-c r e / E s r 1^{*}\right)^{1 V c o}$ ) or TAM animals that received the same tamoxifen treatment but were not carrying the Cre transgene and therefore retained a functional Ncaph $2^{f l o x}$ fflox genotype. (C) Expression level of Ncaph2 in hepatocytes. Each dot shows an independent measurement of normalized RT-qPCR expression. Whiskers indicate standard deviation. Expression levels of Ncaph2 in TAM and $\triangle \mathrm{Ncaph} 2$ are significantly different (TAM: $1.000 \pm 0.1887$ s.e.m.; $\Delta$ Ncaph2: $0.05603 \pm 0.005896, n=7 ; p$-value $=0.0003$ (unpaired two tailed $t$-test). (D) RNA-Seq tracks on the Ncaph2 locus show deletion of the three floxed exons in $\Delta N$ caph2 hepatocytes. Data pooled from 4 independently processed animals for each group. (E-F) Western blot detection of NCAPH2 in $\triangle$ Ncaph2 and control samples. (G) Hi-C maps of chromosome 11 across four conditions with compartment eigenvector tracks plotted above. From left to right: WT, TAM, $\Delta$ Ncaph2 and $\Delta$ Nipbl. The top row shows whole chromosome maps, the middle row shows a zoom-in of a 30-Mb region, and the bottom row shows a further zoom-in of a 6-Mb region. The color mapping in the top and middle row is logarithmic to highlight differences in compartmentalization, and linear in the bottom row to highlight TAD-associated features. 
bioRxiv preprint doi: https://doi.org/10.1101/437459; this version posted October 7, 2018. The copyright holder for this preprint (which was not certified by peer review) is the author/funder, who has granted bioRxiv a license to display the preprint in perpetuity. It is made available under aCC-BY 4.0 International license.

Figure 2: Condensin II removal in differentiated hepatocytes does not significantly impact interphase chromatin organization

A

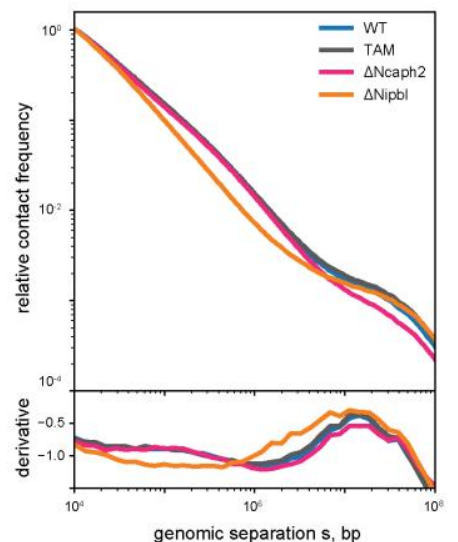

$\mathrm{E}$

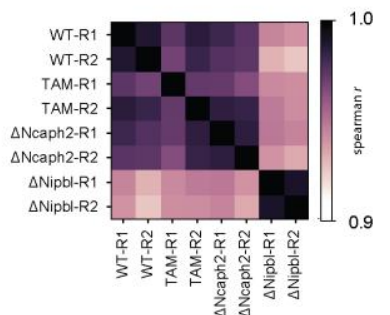

G

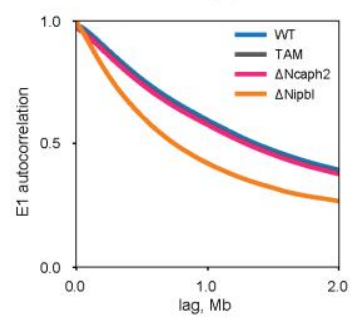

I
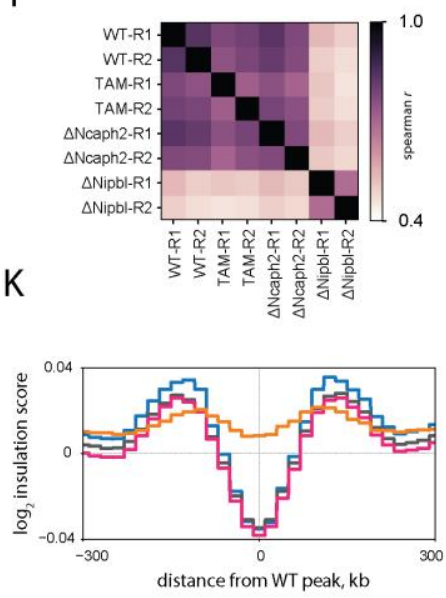

B

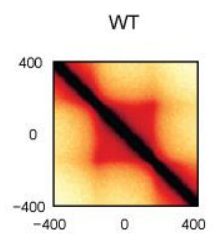

C

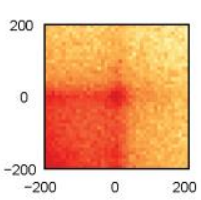

D
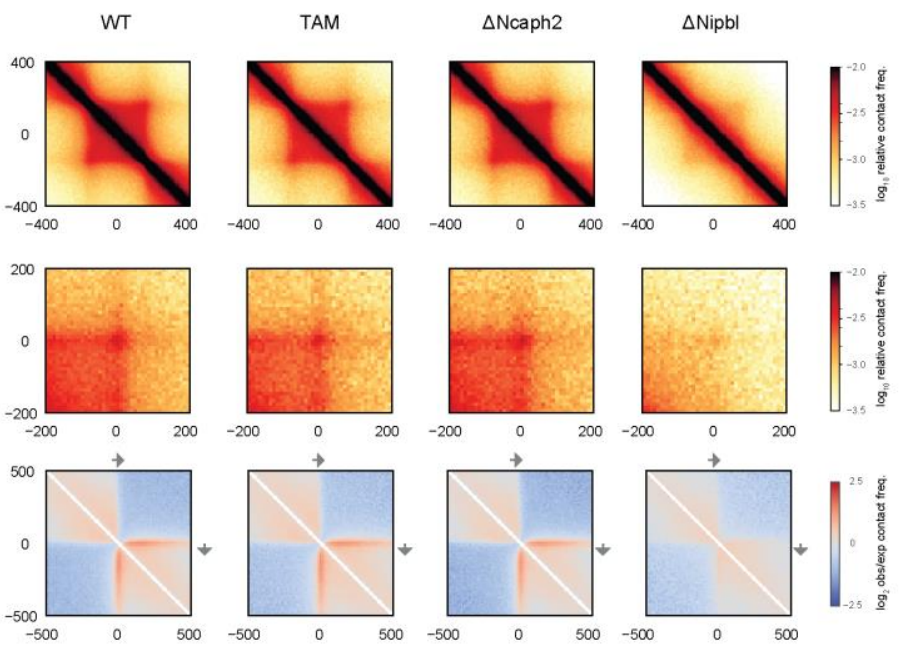

F
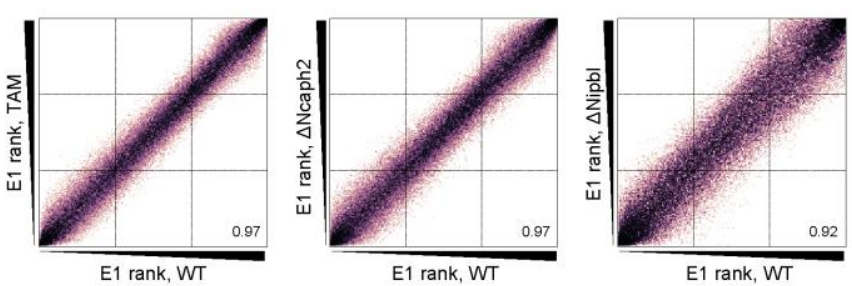

$\mathrm{H}$
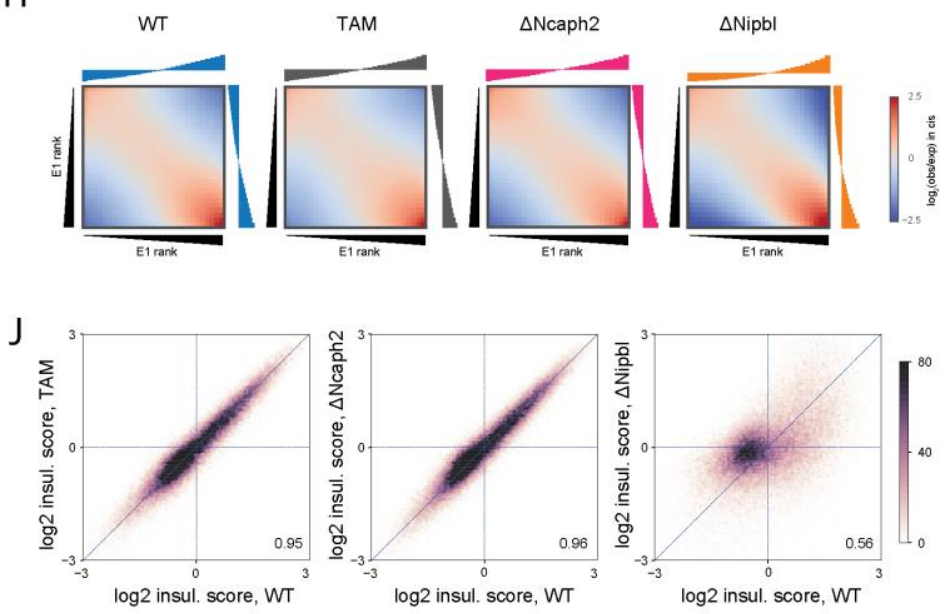

L
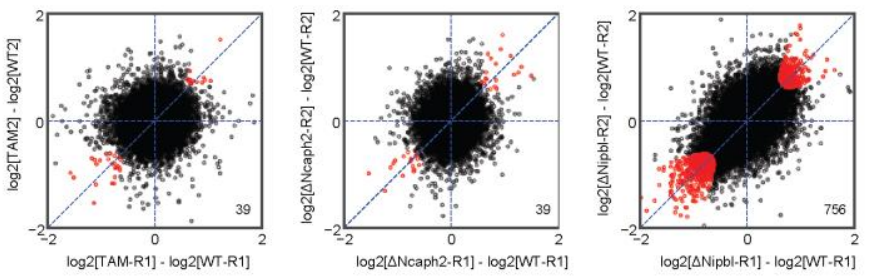
(A) Top panel: genome-wide $\mathrm{P}(\mathrm{s})$ contact frequency curves in WT, TAM control, $\triangle \mathrm{Ncaph} 2$ and $\triangle$ Nipbl. Bottom panel: derivatives of P(s) curves. (B, C, D) Average Hi-C maps centered at (B) WT domains of length $300-400 \mathrm{~kb}$, (C) $102 \mathrm{Hi}-\mathrm{C}$ peaks with separation 500-600kb, (D) 20,000 CTCF binding sites detected in WT from (Schwarzer et al., 2017), oriented by the core CTCF motif. (E) Heatmap of Spearman correlation coefficients of compartment tracks among replicates. (F) Rank-based scatter plots of compartment eigenvectors between pairs of conditions: TAM, $\Delta$ Ncaph2, and $\Delta \mathrm{Nipbl}$ versus WT at $20-\mathrm{kb}$ resolution. $r$ values inset. (G) Genome-wide autocorrelation function of the compartment eigenvectors. (H) "Saddle" heatmap plots of compartment signal for all four conditions. Each element of the heatmap contains the average observed-over-expected contact frequency between pairs of loci (20-kb bins) arranged by rank of their compartment score (E1 rank) and grouped into 30 quantiles. The histograms along the top and right axes show the median compartment scores associated with each quantile of genomic bins. (I) Heatmap of Spearman correlation coefficients of log2 insulation score tracks among replicates. (J) Scatter plots of log2 diamond insulation score between pairs of conditions: TAM, $\Delta$ Ncaph2, and $\Delta$ Nipbl versus WT at $20-\mathrm{kb}$ resolution. Spearman $r$ values inset. (K) Profiles of average insulation score at called insulating loci called in WT. (L) Scatter plots of $\log _{2}$ fold change in insulation score for each of TAM, $\Delta$ Ncaph2, and $\Delta$ Nipbl relative to WT at loci determined to be insulating in at least one condition. Points corresponding to significant differentially insulated loci are colored red. 
Figure 3: Condensin II removal does not influence specific interactions in the Hist3 gene locus, nor those between transcriptionally active or insulating genomic landmarks

A

WT

$\Delta$ Ncaph2

$\Delta$ Nipbl
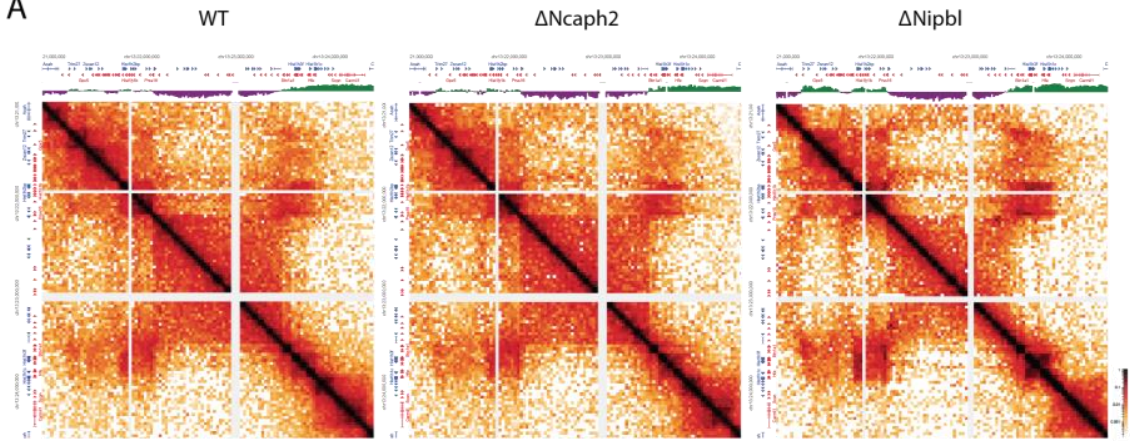

B

WT

TAM

$\Delta$ Ncaph2

$\Delta \mathrm{Nipbl}$
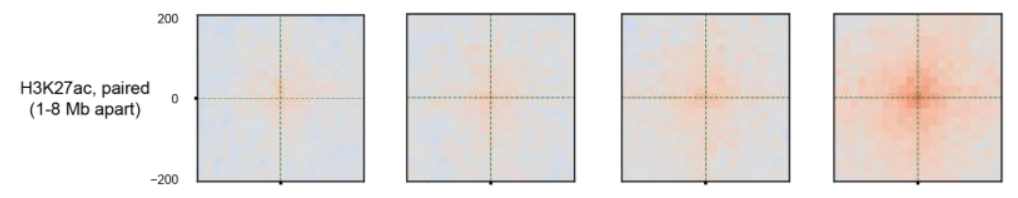

C
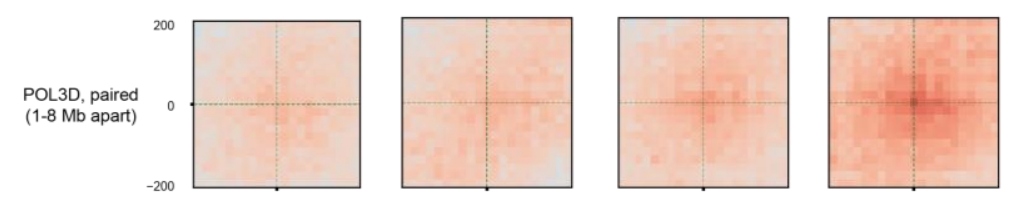

D
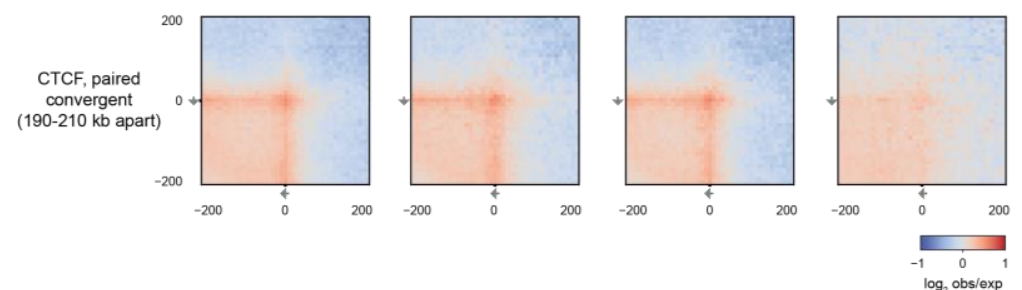

$\log _{2}$ obs/exp
contact frequency

(A) Hi-C maps of the histone gene cluster locus on mouse chromosome 13 in WT (left), $\Delta$ Ncaph2 (middle) and $\Delta \mathrm{Nipbl}$ (right) displayed in HiGlass (Kerpedjiev et al., 2018). Gene annotations and compartment eigenvector tracks are displayed along the borders. (B, C) Average observed/expected $\mathrm{Hi}-\mathrm{C}$ pileup maps around the interaction points of paired H3K27ac peaks and Pol3 peaks with separations in the range of 1-8 $\mathrm{Mb}$ in cis. (D) Average observed/expected $\mathrm{Hi}-\mathrm{C}$ pileup maps around the interaction points of paired CTCF sites with separations of 190-210 $\mathrm{kb}$ in cis. 
Figure 4: Condensin II removal has negligible effects on interphase gene expression

A

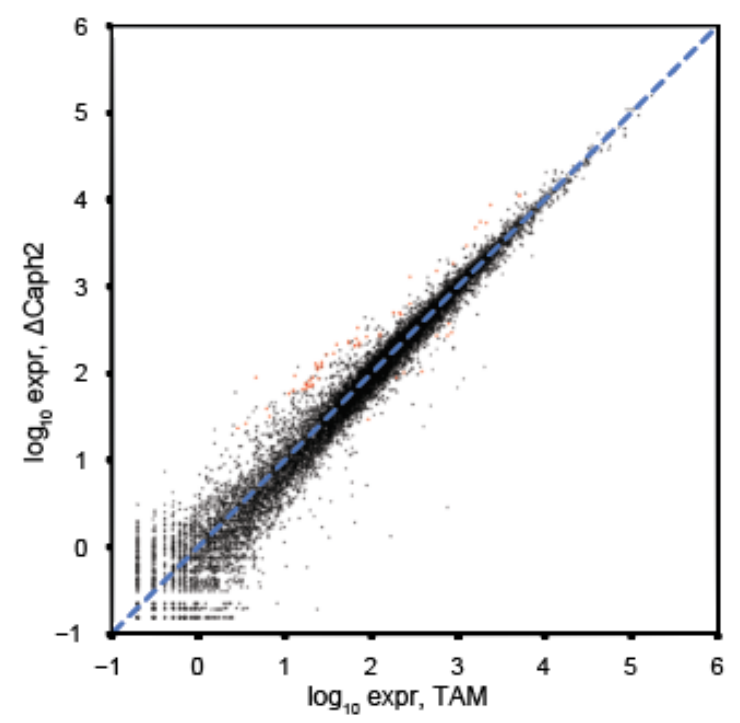

B

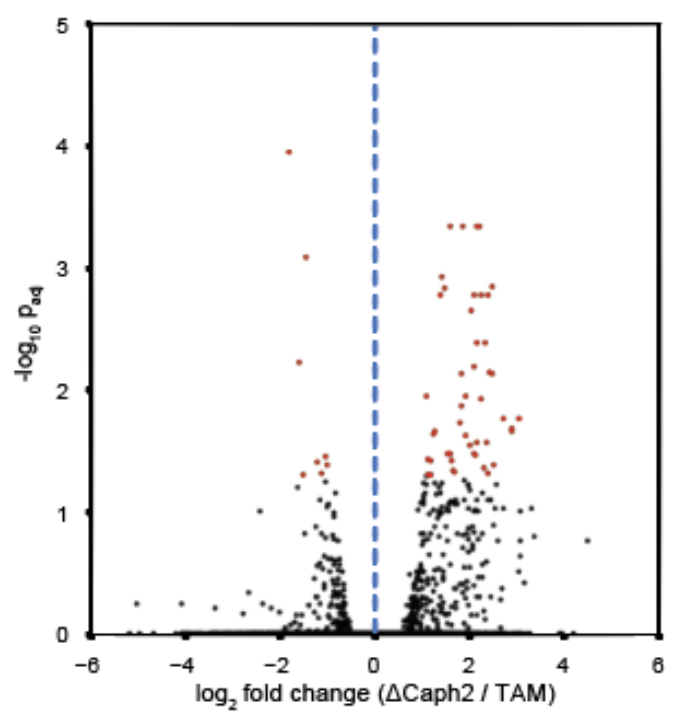

(A) Scatter plot of log expression (read counts normalized for sequence depth) between TAM and $\triangle$ Ncaph2 conditions. (B) Volcano plot of differential expression analysis. Significantly differentially expressed genes at a false discovery rate of $\leq 0.05$ are colored in red. 
Figure 5: Condensin II degradation in synchronized DT40 chicken cells reveals severe impact on mitotic chromosome folding but no effect during $\mathbf{G 2}$

A

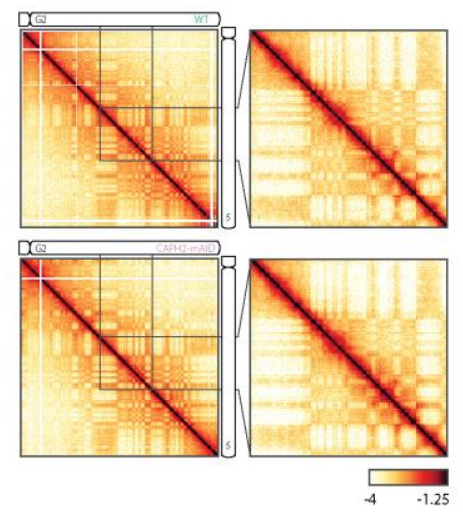

B

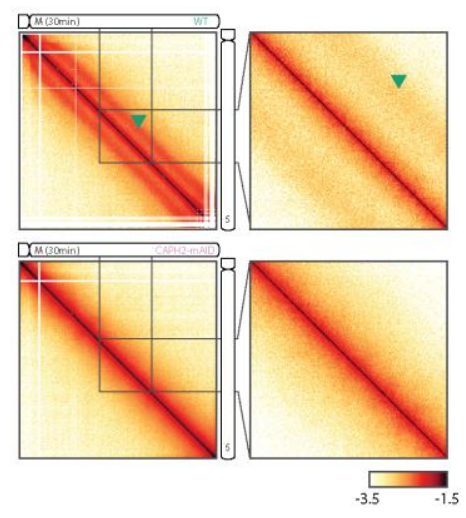

C

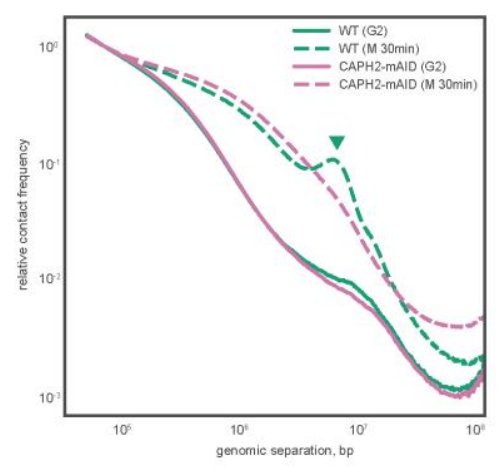

(A) Hi-C map of chromosome 5 in G2-arrested DK40 chicken cells in WT, above, and AIDmediated degradation of $\mathrm{CAPH} 2$, below. (B) Hi-C map of same regions in late prometaphase ( $\mathrm{t}=30 \mathrm{~min}$ ). Top: WT, Bottom: $\mathrm{CAPH} 2$ depletion. (C) Genome-wide $P(s)$ curves for the conditions in $A$ and $B$, at both cell cycle stages. Data re-analyzed from Gibcus et al, 2018. Note the disappearance of the "bump" in the $P(s)$ curve in the late prometaphase CAPH2 depletion condition (dashed curves). On $\mathrm{HiC}$ maps from cells in late prometaphase, this bump corresponds to a second diagonal band (green arrows in B), which disappears in the CAPH2 depletion condition. This is interaction pattern is indicative of a condensin II-dependent regular helical winding of a condensed chromatin scaffold (Gibcus et al, 2018). By contrast, CAPH2 depletion does not significantly affect $P(s)$ or Hi-C maps in G2-arrested DK40 cells (solid curves in $\mathbf{C}, \mathrm{Hi}-\mathrm{C}$ maps in $\mathbf{A}$ ). 
bioRxiv preprint doi: https://doi.org/10.1101/437459; this version posted October 7, 2018. The copyright holder for this preprint (which was not certified by peer review) is the author/funder, who has granted bioRxiv a license to display the preprint in perpetuity. It is made available under aCC-BY 4.0 International license.

Table 1 - Differentially expressed genes in $\Delta$ Ncaph2 samples

\begin{tabular}{|c|c|c|c|c|c|}
\hline Symbol & $\begin{array}{c}\text { foldChange } \\
\Delta \mathrm{NcapH2} \text { vs WT }\end{array}$ & P.val & P.adj (FDR<5\%) & $\begin{array}{c}\text { Circadian Peak (ZT) and } \\
\text { amplitude }\end{array}$ & Cell Cycle \\
\hline Nr1d1 & 0.28 & $2.04 \mathrm{E}-08$ & $1.13 \mathrm{E}-04$ & ZT $7 ; 6.5$ & \\
\hline Bhlhe41 & 0.33 & $5.64 \mathrm{E}-06$ & 5.97E-03 & ZT 8; n.d. & \\
\hline Rnf152 & 0.34 & 1.43E-04 & $5.00 \mathrm{E}-02$ & ZT $6 ; 1.4$ & \\
\hline Fgfr2 & 0.36 & $3.33 \mathrm{E}-07$ & $8.21 \mathrm{E}-04$ & ZT $7 ; 0.5$ & \\
\hline Rgs3 & 0.42 & $8.95 \mathrm{E}-05$ & $3.90 \mathrm{E}-02$ & & \\
\hline KIhdc7a & 0.42 & 4.99E-06 & $5.55 \mathrm{E}-03$ & & \\
\hline$T b c 1 d 30$ & 0.45 & $1.30 \mathrm{E}-04$ & 4.91E-02 & ZT $8 ; 0.8$ & \\
\hline Flen & 0.48 & 7.37E-05 & $3.56 \mathrm{E}-02$ & ZT $7 ; 1.1$ & \\
\hline S/c23a2 & 0.49 & 9.94E-05 & 4.17E-02 & ZT $6 ; 0.8$ & \\
\hline Esr1 * & 2.11 & $1.37 \mathrm{E}-05$ & $1.13 \mathrm{E}-02$ & ZT $18 ; 1$ & \\
\hline Ints2 & 2.13 & $1.34 \mathrm{E}-04$ & 4.97E-02 & ZT $19 ; 1$ & \\
\hline Herpud1 & 2.15 & 7.84E-05 & $3.71 \mathrm{E}-02$ & ZT $11 ; 1.3$ & \\
\hline Nop58 & 2.21 & $1.44 \mathrm{E}-04$ & $5.00 \mathrm{E}-02$ & ZT $17 ; 0.5$ & \\
\hline Rbbp8 & 2.22 & $8.68 \mathrm{E}-05$ & $3.86 \mathrm{E}-02$ & ZT $14 ; 0.5$ & $* \mathrm{G} 1 / \mathrm{S}$ \\
\hline Baiap2 & 2.23 & $1.40 \mathrm{E}-04$ & $5.00 \mathrm{E}-02$ & ZT $19 ; 0.7$ & \\
\hline Epha2 & 2.32 & $3.72 \mathrm{E}-05$ & $2.30 \mathrm{E}-02$ & & \\
\hline St3gal5 & 2.36 & $3.36 \mathrm{E}-05$ & $2.20 \mathrm{E}-02$ & ZT $13 ; 1.9$ & \\
\hline Cyp2a5 & 2.56 & $1.21 \mathrm{E}-06$ & $1.69 \mathrm{E}-03$ & ZT $14 ; 2.3$ & \\
\hline Zfp9 & 2.65 & 5.89E-05 & $3.19 \mathrm{E}-02$ & & \\
\hline Mme & 2.65 & $5.39 \mathrm{E}-07$ & $1.20 \mathrm{E}-03$ & & \\
\hline Arrdc2 & 2.75 & 7.94E-07 & $1.47 \mathrm{E}-03$ & ZT 9;0.6 & \\
\hline Mcm4 & 2.84 & $6.64 \mathrm{E}-05$ & $3.37 \mathrm{E}-02$ & ZT $19 ; 0.8$ & $* M$ \\
\hline Tsku & 2.97 & $1.53 \mathrm{E}-07$ & 4.64E-04 & ZT $15 ; 2.9$ & \\
\hline Nqo1 & 2.99 & $6.47 \mathrm{E}-05$ & 3.37E-02 & ZT $15 ; 1.1$ & \\
\hline Ppp1r3b & 3.06 & $8.47 \mathrm{E}-05$ & $3.84 \mathrm{E}-02$ & ZT $18 ; 2.5$ & \\
\hline Pole & 3.12 & $1.13 \mathrm{E}-04$ & $4.59 \mathrm{E}-02$ & & * \\
\hline Nek2 & 3.13 & $1.18 \mathrm{E}-04$ & $4.69 \mathrm{E}-02$ & & $* M$ \\
\hline An/n & 3.43 & $2.67 \mathrm{E}-05$ & $1.86 \mathrm{E}-02$ & & ${ }^{*} \mathrm{M}$ \\
\hline Plk4 & 3.48 & $1.80 \mathrm{E}-05$ & $1.38 \mathrm{E}-02$ & & \\
\hline 4632434I11Rik & 3.48 & $8.33 \mathrm{E}-06$ & 7.41E-03 & & $*$ \\
\hline Lpin1 & 3.57 & $1.67 \mathrm{E}-07$ & $4.64 \mathrm{E}-04$ & ZT $12 ; 3.2$ & \\
\hline Tmc7 & 3.70 & $1.33 \mathrm{E}-05$ & $1.13 \mathrm{E}-02$ & ZT $14 ; 1.3$ & \\
\hline Tacc3 & 3.76 & 3.91E-05 & $2.35 \mathrm{E}-02$ & & $* \mathrm{G} 2 / \mathrm{M}$ \\
\hline Cdca2 & 3.94 & $5.15 \mathrm{E}-05$ & $2.86 \mathrm{E}-02$ & ZT 18; 1 & $* \mathrm{G} 2$ \\
\hline Hist1h1b & 4.04 & 1.73E-06 & $2.26 \mathrm{E}-03$ & & \\
\hline Fign/1 & 4.18 & $1.20 \mathrm{E}-06$ & $1.69 \mathrm{E}-03$ & & \\
\hline Ect2 & 4.18 & $6.68 \mathrm{E}-05$ & $3.37 \mathrm{E}-02$ & & $* M$ \\
\hline Zwilch & 4.21 & $6.46 \mathrm{E}-06$ & $6.52 \mathrm{E}-03$ & ZT $15 ; 0.8$ & $*$ \\
\hline Kif20a & 4.29 & $7.02 \mathrm{E}-05$ & $3.46 \mathrm{E}-02$ & & $*$ kinesin \\
\hline Cdca3 & 4.37 & $3.57 \mathrm{E}-06$ & 4.17E-03 & & $* \mathrm{G} 2 / \mathrm{M}$ \\
\hline Dtl & 4.38 & $1.34 \mathrm{E}-07$ & 4.64E-04 & ZT $17 ; 1.1$ & G1 \\
\hline Kif4 & 4.42 & $4.68 \mathrm{E}-05$ & $2.72 \mathrm{E}-02$ & & $*$ kinesin \\
\hline Kif $20 b$ & 4.59 & $1.08 \mathrm{E}-07$ & $4.64 \mathrm{E}-04$ & & * kinesin G2/M \\
\hline$M f s d 2 a$ & 4.63 & $1.05 \mathrm{E}-06$ & $1.67 \mathrm{E}-03$ & ZT $13 ; 5.2$ & \\
\hline Ddit4 & 4.72 & $1.50 \mathrm{E}-05$ & 1.19E-02 & ZT $18 ; 1.6$ & \\
\hline E130306D19Rik & 4.82 & $1.06 \mathrm{E}-04$ & $4.35 \mathrm{E}-02$ & & \\
\hline Plin4 & 4.84 & 4.27E-11 & $3.16 \mathrm{E}-07$ & ZT $15 ; 1.4$ & \\
\hline Cdc45 & 4.94 & $3.33 \mathrm{E}-06$ & $4.11 \mathrm{E}-03$ & ZT $15 ; 0.9$ & $*$ \\
\hline Ckap2 & 5.03 & $4.78 \mathrm{E}-05$ & $2.72 \mathrm{E}-02$ & & $* \mathrm{G} 2 / \mathrm{M}$ \\
\hline$T p \times 2$ & 5.14 & $1.26 \mathrm{E}-04$ & $4.83 \mathrm{E}-02$ & & $* M$ \\
\hline Dlgap5 & 5.15 & $9.90 \mathrm{E}-07$ & $1.67 \mathrm{E}-03$ & & ${ }^{*} \mathrm{M}$ \\
\hline Mastl & 5.30 & $7.45 \mathrm{E}-06$ & 7.20E-03 & & $* S$ \\
\hline Zbtb16 & 5.47 & $7.55 \mathrm{E}-16$ & $1.50 \mathrm{E}-11$ & ZT $14 ; 1.3$ & \\
\hline Gstm3 & 5.48 & $7.08 \mathrm{E}-07$ & $1.43 \mathrm{E}-03$ & ZT $19 ; 0.6$ & \\
\hline D2Ertd750e & 5.56 & $8.12 \mathrm{E}-06$ & $7.41 \mathrm{E}-03$ & & \\
\hline Cenpe & 5.58 & $9.83 \mathrm{E}-05$ & $4.17 \mathrm{E}-02$ & & ${ }^{*}$ kinetochore $\mathrm{M}$ \\
\hline D17H6S56E-5 & 5.96 & $1.42 \mathrm{E}-04$ & $5.00 \mathrm{E}-02$ & & \\
\hline
\end{tabular}


bioRxiv preprint doi: https://doi.org/10.1101/437459; this version posted October 7, 2018. The copyright holder for this preprint (which was not certified by peer review) is the author/funder, who has granted bioRxiv a license to display the preprint in perpetuity. It is made available under aCC-BY 4.0 International license.

\begin{tabular}{|cccccc|}
\hline Gm16090 & 6.43 & $8.40 \mathrm{E}-05$ & $3.84 \mathrm{E}-02$ & & \\
\hline$P b k$ & 6.45 & $2.44 \mathrm{E}-05$ & $1.75 \mathrm{E}-02$ & & $* \mathrm{G} 2 / \mathrm{M}$ \\
\hline Kntc1 & 7.26 & $3.08 \mathrm{E}-05$ & $2.08 \mathrm{E}-02$ & ZT $18 ; 1.3$ & \\
\hline Rad51ap1 & 7.39 & $3.46 \mathrm{E}-05$ & $2.20 \mathrm{E}-02$ & & $\mathrm{~S}$ \\
\hline Asf1b & 8.20 & $2.45 \mathrm{E}-05$ & $1.75 \mathrm{E}-02$ & ZT 21; 3.4 & $*$ \\
\hline Cdkn1a & 19.10 & $1.35 \mathrm{E}-15$ & $1.50 \mathrm{E}-11$ & ZT $22-$ n.d. & \\
\hline Gsta1 & 19.16 & $1.23 \mathrm{E}-04$ & $4.80 \mathrm{E}-02$ & & \\
\hline
\end{tabular}

Peak time and amplitude of circadian variation were taken from Wang et al. 2018. Cell cycle data was obtained from Cyclebase v3 (Santos et al. 2015)

Table 2 - Molecular and cellular processes identified by GO-term enrichment analysis.

\begin{tabular}{|l|l|l|l|l|}
\hline G0 term & Description & P-value & FDR q-value & Enrichment \\
\hline G0:0007049 & cell cycle & $1.38 \mathrm{E}-14$ & $2.04 \mathrm{E}-10$ & $7.79(22)$ \\
\hline G0:1903047 & $\begin{array}{l}\text { mitotic cell cycle } \\
\text { process }\end{array}$ & $3.07 \mathrm{E}-13$ & $2.28 \mathrm{E}-09$ & $10.29(17)$ \\
\hline G0:0022402 & cell cycle process & $1.02 \mathrm{E}-12$ & $5.03 \mathrm{E}-09$ & $7.29(20)$ \\
\hline G0:0051301 & cell division & $2.98 \mathrm{E}-12$ & $1.10 \mathrm{E}-08$ & $9.92(16)$ \\
\hline G0:0051988 & $\begin{array}{l}\text { regulation of } \\
\text { attachment of spindle } \\
\text { microtubules to } \\
\text { kinetochore }\end{array}$ & $7.73 \mathrm{E}-08$ & $1.43 \mathrm{E}-04$ & $90.21(4)$ \\
\hline G0:0007051 & spindle organization & $7.77 \mathrm{E}-6$ & $9.6 \mathrm{E}-3$ & $12.61(6)$ \\
\hline
\end{tabular}




\section{Material and methods}

\section{Mouse lines}

B6N;B6N-Ncaph2 ${ }^{<\mathrm{tm} 1 \mathrm{a}(\mathrm{EUCOMM}) W \mathrm{tsi} / \text { Wtsi }}$ mice (thereafter referred as Ncaph2 ${ }^{\text {lox/ }+}$ ) mice were generously provided by WTSI and maintained on C57BL/6J genetic background.

For deletion of the floxed exon and generation of $\Delta$ Ncaph2 hepatocytes, Ncaph2 lox/t+ were bred to Ttr-cre/Esr1 (Tannour-Louet et al., 2002) transgenic mice. 12 week-old mice were injected with $1 \mathrm{mg}$ Tamoxifen $(100 \mu \mathrm{l}$ of $10 \mathrm{mg} / \mathrm{ml}$ Tamoxifen in corn oil) on 5 consecutive days. After another 5 days without injection, these mice were sacrificed and the hepatocytes were harvested as previously described (Schwarzer et al., 2017). Mice were genotyped by PCR using specific primer pairs (details available on request). Mouse experiments were conducted in accordance with the principles and guidelines in place at European Molecular Biology Laboratory, as defined and overseen by its Institutional Animal Care and Use Committee, in accordance with the European Directive 2010/63/EU.

\section{Western blots}

Nuclear proteins from tamoxifen-treated wild-type C57BL/6J (TAM) and $\Delta$ Ncaph2 hepatocytes

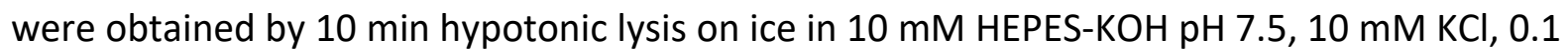
mM EDTA, 0.5\% v/v NP-40, 1 mM DTT, 0.5 mM PMSF and Roche Complete protease inhibitor cocktail, followed by collection of the nuclei $(5 \mathrm{~min}$ at $1000 \mathrm{~g}$ ) and protein extraction in same buffer supplemented with $1 \% \mathrm{w} / \mathrm{v}$ SDS and $150 \mathrm{mM} \mathrm{NaCl}$ at $65^{\circ} \mathrm{C}$ for $10 \mathrm{~min}$. Total protein concentrations were estimated by Bradford assay (Biorad) and $11 \mu \mathrm{g}$ protein was resolved by SDS-PAGE on $10 \%$ polyacrylamide or 3-8\% NuPAGE Tris-Acetate (Thermo Fisher) gels and transferred to nitrocellulose. Membranes were pretreated and probed in PBS, $0.1 \% \mathrm{v} / \mathrm{v}$ Tween$20,4 \% \mathrm{w} / \mathrm{v}$ non-fat milk powder with rabbit primary antibodies and goat anti-rabbit IgG-HRP (Dianova) secondary antibody.

As commercial antibodies raised against fragments of human NCAPH2 (Bethyl A302-275A; Abcam 83848) did not detect protein in the expected range, we raised a rabbit polyclonal antibody against full-length mouse NCAPH2. The antibody recognized multiple proteins from the mouse hepatocyte nuclear extracts, including a $105 \mathrm{kDa}$ protein that was depleted in $\triangle \mathrm{Ncaph} 2$ mice as well as human NCAPH2 in HeLa whole cell extracts.

\section{RT-qPCR}

Unfixed hepatocyte aliquots were thawed and RNA was prepared with Qiagen RNeasy Kit. cDNA was generated using NEB ProtoScript ${ }^{\circledR}$ First Strand cDNA Synthesis Kit with random primer mix. RT-qPCR was performed with Applied SYBR Green PCR Master Mix and following primers: Ncaph2-ex8/9-qPCR_F ACGGGAGTCCTGTTCCTGTA, Ncaph2-ex8/9-qPCR_R CTCTGCATCCTCCTCTCCAC, Gapdh-qPCR_F CTCCCACTCTTCCACCTTCG， Gapdh-qPCR_R 


\section{RNA-seq libraries and sequencing}

Unfixed hepatocyte aliquots were thawed and RNA was prepared with Qiagen RNeasy Kit. RNA integrity was tested with Bioanalyzer (Agilent RNA Nano Kit) and ribosomal RNA was removed using Ribo-Zero rRNA Removal Kit (Illumina) prior to library preparation. Strand-specific libraries were prepared with NEBNext ${ }^{\circledR}$ Ultra ${ }^{\mathrm{TM}}$ Directional RNA Library Prep Kit for Illumina ${ }^{\circledR}$. After amplification and size selection with Agencourt AMPure XP beads (Beckmann Coulter) their size-distributions were determined with Bioanalyzer. Equimolar pools of libraries were sequenced with Illumina HiSeq2000 (50bp, single end). Reads were uniquely mapped to the reference genome $\mathrm{NCBI} 37 / \mathrm{mm}$.

\section{Tethered Chromatin Capture (TCC)}

Roughly 100 mio fixed hepatocytes per sample were processed according to Kalhor et al. (Kalhor et al., 2012) using HindIII. Libraries were PCR-amplified (12 cycles) and size selected with $\mathrm{E}-G e l^{\circledR}$ SizeSelect ${ }^{\mathrm{TM}}$ (Thermo Fisher). Equimolar pools of libraries were sequenced with Illumina HiSeq2000 (50bp, paired end). We retrieved between 100 and 150 mio paired reads per sample, of which $\sim 40 \%$ had both sides uniquely mapped to the reference genome $\mathrm{NCBI} 37 / \mathrm{mm} 9$.

\section{Computational Analysis}

\section{Hi-C data processing}

$\mathrm{Hi}-\mathrm{C}$ datasets were processed using a portable pipeline driven by the nextflow workflow manager ( $\mathrm{Di}$ Tommaso et al., 2017) called distiller, available at https://github.com/mirnylab/distiller-nf. Briefly, we mapped Hi-C sequencing reads to the mouse reference assembly mm9 using bwa mem (Li 2013) with flags -SP. Alignments were parsed, filtered for duplicates and pairs were classified using the pairtools package (https://github.com/mirnylab/pairtools). Hi-C pairs were aggregated into contact matrices in the cooler format using the cooler package (https://github.com/mirnylab/cooler) at multiple resolutions. All contact matrices were normalized using the iterative correction procedure (Imakaev et al., 2012) after bin-level filtering. Low-coverage bins were excluded using the MADmax (maximum allowed median absolute deviation) filter on genomic coverage, described in (Schwarzer et al., 2017), using a threshold of 5.0 MADs. To remove short-range Hi-C artifactssuch as unligated and self-ligated $\mathrm{Hi}-\mathrm{C}$ molecules-we removed contacts mapping to the same or adjacent genomic bins, up to a distance of at least $10 \mathrm{~kb}$. The same procedure was used to process the DT40 Hi-C data sets from (Gibcus et al., 2018). Expected contact profiles were obtained by dividing each diagonal of a cis contact matrice by its average value over nonfiltered genomic bins. This was performed using the cooltools package (https://github.com/mirnylab/cooltools). 


\section{$P(s)$ curves}

Scaling curves of contact frequency $P$ as a function of genomic separation $s$ were generated using cooltools by aggregating normalized contact frequency over valid pixels along diagonals of $1 \mathrm{~kb}$-resolution cis contact maps, with diagonals grouped into geometrically increasing spans of genomic separation. Average contact frequency $\mathrm{P}(\mathrm{s})$ curves are displayed using log-log axes.

\section{Compartmentalization analysis}

Compartmentalization as reflected in $\mathrm{Hi}-\mathrm{C}$ maps was quantified using an eigenvector decomposition procedure based on (Imakaev et al., 2012), as implemented in the cooltools package. In short, compartment tracks (that is, the values or scores of compartment signal across all genomic bins) were quantified as the dominant eigenvector of the observed or expected $20-\mathrm{kb}$ and $100-\mathrm{kb}$ cis contacts maps upon subtraction of 1.0 and rescaling by the magnitude of the square root of its eigenvalue.

Compartmentalization strength was assessed by taking the average contact enrichment between pairs of loci with whose eigenvector scores lied in the extremes of the distribution of eigenvector scores and computing the ratio $(A A+B B) /(2 * A B)$, where $A A$ refers to the average enrichment of loci whose two bins both lie in the top $n \%$ of eigenvector scores, $B B$ to that of loci whose two bins both lie in the bottom $n \%$ of eigenvector scores, and $A B$ to that of loci whose two bins have strong $A$ and $B$ compartment signal, respectively. The fraction at $n=20 \%$ was used as an overall measure of compartmentalization strength. Saddle plot heatmaps and compartmentalization strength profiles were created using the cooltools package.

\section{Domain detection and peak coordinates}

We used the domains detected in the WT condition using in the lavaburst package (https://github.com/nvictus/lavaburst) from (Schwarzer et al., 2017). While domain callers do not distinguish compartmental domains from TADs, the majority of domains identified in the WT condition were shown to correspond to TADs. To characterize the structure of known $\mathrm{Hi}-\mathrm{C}$ peaks in our data, we used the list of peaks detected in $\mathrm{Hi}-\mathrm{C}$ maps for the $\mathrm{CH} 12-\mathrm{LX}$ mouse cell line (Rao et al., 2014).

\section{Insulation analysis}

Diamond insulation scores (Crane et al., 2015) were calculated on all 20-kb and 40-kb maps using the cooltools package. Additionally, an insulation minima calling procedure based on peak prominence, described in (Nora et al., 2017) was used to define insulating loci. We considered only insulating loci called in both replicate $\mathrm{Hi}-\mathrm{C}$ maps and in the pooled $\mathrm{Hi}-\mathrm{C}$ maps for each condition, at $20-\mathrm{kb}$ resolution. Differential insulation analysis was performed using quantilenormalized $\log _{2}$ insulation scores on $\sim 11,000$ loci classified as insulating in at least one of the four conditions. Since each pair of treatment-control conditions considered (WT vs TAM, WT vs $\Delta$ Ncaph2, WT vs $\Delta$ Nipbl) consisted of two replicates each, we compared the $\log _{2}$ ratios of insulation score between conditions (treatment vs control) and repeated the same procedure on ratios within the same condition (replicate-1 vs replicate-2). Because our sample size for hypothesis testing on each locus was small $(n=2)$, we use the regularized t-test (Baldi and Long, 
2001; Cui and Churchill, 2003) that combines information from the locus-specific variance in log-ratio in with a background variance estimate obtained from the bulk of insulating sites considered. We use a positive false discovery rate (Storey, 2003) of 0.05 on all three sets of hypothesis combined to set a common significance threshold.

\section{ChIP-seq processing and H3K27ac clustering}

We used H3K27ac ChIP-seq peaks and motif assignments from (Schwarzer et al., 2017). To identify regions that exhibit enhancer characteristics, we followed the H3K27ac peak-stitching strategy using a $12.5 \mathrm{~kb}$ stitching distance as in (Hnisz et al., 2013). Rather than defining a cutoff for super-enhancer status, we divided the stitched regions into equal sized groups, first by length, then by expression level as quantified by RNA-seq coverage in WT from (Schwarzer et al., 2017). Additionally, we obtained a curated list of super-enhancers for mouse liver from dbSuper (Khan and Zhang, 2016). ChIP-seq data from (Canella et al., 2012)and (Yuen et al., 2017) were reprocessed following the steps of the ENCODE ChIP-seq pipeline (https://github.com/ENCODEDCC/chip-seq-pipeline). Peaks were also divided into two equal sized groups by expression level.

\section{Hi-C pileup maps}

We used cooltools to calculate aggregate iteratively corrected or observed-over-expected contact frequency maps (pileup maps) centered at specific genomic loci and bounded by a fixed flanking genomic distance. For single genomic landmarks or intervals (TAD/peak calls, CTCF sites), pileup maps are centered on the main diagonal at each feature's midpoint. For the $\mathrm{Hi}-\mathrm{C}$ pileup maps of paired genomic loci centered away from the $\mathrm{Hi}-\mathrm{C}$ map's main diagonal, we selected 5000 random pairs without replacement in trans and for each range of genomic distances in cis from each set of landmark features. We then used cooltools to calculate observed-over-expected $\mathrm{Hi}-\mathrm{C}$ pileup maps centered at the midpoint coordinates of the landmark feature pairs.

\section{RNA-seq and GO enrichment analysis}

We mapped the RNA-seq data to the $\mathrm{mm} 9$ reference mouse genome assembly and GENCODE VM1 transcriptome using STAR v.2.5.0a60 (Dobin et al., 2013). To obtain the tracks of local transcription, we aggregated the uniquely mapped reads into RPM-normalized bigWig files using the built-in STAR functionality. To find differentially expressed genes, we aggregated the read counts at the gene level using HTSeq (Anders et al., 2015) with the 'union' option and called differentially expressed genes with DESeq2 (Love et al., 2014).

\section{Circadian rhythm and cell cycle data analysis}

We used the data from (Wang et al., 2018), except for Gsta1 (Zhang et al., 2009) and Bhlhe41 (Tognini et al., 2017). Cell cycle information was inferred from (Santos et al., 2015). 


\section{REFERENCES}

Anders, S., Pyl, P.T., and Huber, W. (2015). HTSeq--a Python framework to work with highthroughput sequencing data. Bioinformatics 31,166-169.

Baldi, P., and Long, A.D. (2001). A Bayesian framework for the analysis of microarray expression data: regularized t-test and statistical inferences of gene changes.

Bioinformatics 17, 509-519.

Bauer, C.R., Hartl, T.A., and Bosco, G. (2012). Condensin II promotes the formation of chromosome territories by inducing axial compaction of polyploid interphase chromosomes. PLoS Genet 8, e1002873.

Canella, D., Bernasconi, D., Gilardi, F., LeMartelot, G., Praz, V., Cousin, P., Delorenzi, M., Hernandez, N., CycliX Consortium, Deplancke, B., et al. (2012). A multiplicity of factors contributes to selective RNA polymerase III occupancy of a subset of RNA polymerase III genes in mouse liver. Genome Res 22, 666-680.

Coronado, D., Godet, M., Bourillot, P.-Y., Tapponnier, Y., Bernat, A., Petit, M., Afanassieff, M., Markossian, S., Malashicheva, A., Iacone, R., et al. (2013). A short G1 phase is an intrinsic determinant of naïve embryonic stem cell pluripotency. Stem Cell Res 10,118-131.

Cos, S., Recio, J., and Sánchez-Barceló, E.J. (1996). Modulation of the length of the cell cycle time of MCF-7 human breast cancer cells by melatonin. Life Sci. 58, 811-816.

Crane, E., Bian, Q., McCord, R.P., Lajoie, B.R., Wheeler, B.S., Ralston, E.J., Uzawa, S., Dekker, J., and Meyer, B.J. (2015). Condensin-driven remodelling of X chromosome topology during dosage compensation. Nature 523, 240-244.

Cui, X., and Churchill, G.A. (2003). Statistical tests for differential expression in cDNA microarray experiments. Genome Biol. 4, 210.

Dekker, J., and Mirny, L. (2016). The 3D Genome as Moderator of Chromosomal Communication. Cell 164, 1110-1121.

Di Tommaso, P., Chatzou, M., Floden, E.W., Barja, P.P., Palumbo, E., and Notredame, C. (2017). Nextflow enables reproducible computational workflows. Nat Biotechnol 35, 316319.

Dixon, J.R., Gorkin, D.U., and Ren, B. (2016). Chromatin Domains: The Unit of Chromosome Organization. Mol Cell 62, 668-680.

Dixon, J.R., Selvaraj, S., Yue, F., Kim, A., Li, Y., Shen, Y., Hu, M., Liu, J.S., and Ren, B. (2012). Topological domains in mammalian genomes identified by analysis of chromatin interactions. Nature 485, 376-380. 
Dobin, A., Davis, C.A., Schlesinger, F., Drenkow, J., Zaleski, C., Jha, S., Batut, P., Chaisson, M., and Gingeras, T.R. (2013). STAR: ultrafast universal RNA-seq aligner. Bioinformatics 29, $15-21$.

Dowen, J.M., Bilodeau, S., Orlando, D.A., Hübner, M.R., Abraham, B.J., Spector, D.L., and Young, R.A. (2013). Multiple structural maintenance of chromosome complexes at transcriptional regulatory elements. Stem Cell Reports 1, 371-378.

Falk, M., Feodorova, Y., Naumova, N., Imakaev, M., Lajoie, B.R., Leonhardt, H., Joffe, B., Dekker, J., Fudenberg, G., Solovei, I., et al. (2018). Heterochromatin drives organization of conventional and inverted nuclei. 244038.

Fang, B., Everett, L.J., Jager, J., Briggs, E., Armour, S.M., Feng, D., Roy, A., Gerhart-Hines, Z., Sun, Z., and Lazar, M.A. (2014). Circadian enhancers coordinate multiple phases of rhythmic gene transcription in vivo. Cell 159, 1140-1152.

Fazzio, T.G., and Panning, B. (2010). Condensin complexes regulate mitotic progression and interphase chromatin structure in embryonic stem cells. J Cell Biol 188, 491-503.

Fudenberg, G., Abdennur, N., Imakaev, M., Goloborodko, A., and Mirny, L.A. (2018).

Emerging Evidence of Chromosome Folding by Loop Extrusion. Cold Spring Harb. Symp. Quant. Biol. 034710.

Fudenberg, G., Imakaev, M., Lu, C., Goloborodko, A., Abdennur, N., and Mirny, L.A. (2016). Formation of Chromosomal Domains by Loop Extrusion. Cell Reports 15, 2038-2049.

Ganem, N.J., and Pellman, D. (2012). Linking abnormal mitosis to the acquisition of DNA damage. J Cell Biol 199, 871-881.

Gause, M., Schaaf, C.A., and Dorsett, D. (2008). Cohesin and CTCF: cooperating to control chromosome conformation? Bioessays 30, 715-718.

George, C.M., Bozler, J., Nguyen, H.Q., and Bosco, G. (2014). Condensins are Required for Maintenance of Nuclear Architecture. Cells 3, 865-882.

Gerlich, D., Hirota, T., Koch, B., Peters, J.-M., and Ellenberg, J. (2006). Condensin I stabilizes chromosomes mechanically through a dynamic interaction in live cells. Curr Biol 16, 333344.

Gibcus, J.H., and Dekker, J. (2013). The Hierarchy of the 3D Genome. Mol Cell 49, 773-782.

Gibcus, J.H., Samejima, K., Goloborodko, A., Samejima, I., Naumova, N., Nuebler, J., Kanemaki, M.T., Xie, L., Paulson, J.R., Earnshaw, W.C., et al. (2018). A pathway for mitotic chromosome formation. Science 359, eaao6135. 
Green, L.C., Kalitsis, P., Chang, T.M., Cipetic, M., Kim, J.H., Marshall, O., Turnbull, L., Whitchurch, C.B., Vagnarelli, P., Samejima, K., et al. (2012). Contrasting roles of condensin I and condensin II in mitotic chromosome formation. J Cell Sci 125, 1591-1604.

Haarhuis, J.H.I., van der Weide, R.H., Blomen, V.A., Yáñez-Cuna, J.O., Amendola, M., van Ruiten, M.S., Krijger, P.H.L., Teunissen, H., Medema, R.H., van Steensel, B., et al. (2017). The Cohesin Release Factor WAPL Restricts Chromatin Loop Extension. Cell 169, 693-707.e14.

Hirano, T. (2016). Condensin-Based Chromosome Organization from Bacteria to Vertebrates. Cell 164, 847-857.

Hirota, T. (2004). Distinct functions of condensin I and II in mitotic chromosome assembly. J Cell Sci 117, 6435-6445.

Hnisz, D., Abraham, B.J., Lee, T.I., Lau, A., Saint-André, V., Sigova, A.A., Hoke, H.A., and Young, R.A. (2013). Super-Enhancers in the Control of Cell Identity and Disease. Cell 155, 934-947.

Hocquet, C., Robellet, X., Modolo, L., Sun, X.-M., Burny, C., Cuylen-Haering, S., Toselli, E., Clauder-Münster, S., Steinmetz, L., Haering, C.H., et al. (2018). Condensin controls cellular RNA levels through the accurate segregation of chromosomes instead of directly regulating transcription. bioRxiv 330654.

Imakaev, M., Fudenberg, G., McCord, R.P., Naumova, N., Goloborodko, A., Lajoie, B.R., Dekker, J., and Mirny, L.A. (2012). Iterative correction of Hi-C data reveals hallmarks of chromosome organization. Nat Methods 9, 999-1003.

Jans, J., Gladden, J.M., Ralston, E.J., Pickle, C.S., Michel, A.H., Pferdehirt, R.R., Eisen, M.B., and Meyer, B.J. (2009). A condensin-like dosage compensation complex acts at a distance to control expression throughout the genome. Genes Dev 23, 602-618.

Kalhor, R., Tjong, H., Jayathilaka, N., Alber, F., and Chen, L. (2012). Genome architectures revealed by tethered chromosome conformation capture and population-based modeling. Nat Biotechnol 30, 90-98.

Kerpedjiev, P., Abdennur, N., Lekschas, F., McCallum, C., Dinkla, K., Strobelt, H., Luber, J.M., Ouellette, S.B., Azhir, A., Kumar, N., et al. (2018). HiGlass: web-based visual exploration and analysis of genome interaction maps. Genome Biol. 19, 125.

Khan, A., and Zhang, X. (2016). dbSUPER: a database of super-enhancers in mouse and human genome. Nucleic Acids Res 44, D164-D171.

Kim, J.H., Zhang, T., Wong, N.C., Davidson, N., Maksimovic, J., Oshlack, A., Earnshaw, W.C., Kalitsis, P., and Hudson, D.F. (2013). Condensin I associates with structural and gene regulatory regions in vertebrate chromosomes. Nature Communications 4, 2537. 
Kranz, A.-L., Jiao, C.-Y., Winterkorn, L.H., Albritton, S.E., Kramer, M., and Ercan, S. (2013). Genome-wide analysis of condensin binding in Caenorhabditis elegans. Genome Biol. 14, R112.

Li, W., Hu, Y., Oh, S., Ma, Q., Merkurjev, D., Song, X., Zhou, X., Liu, Z., Tanasa, B., He, X., et al. (2015). Condensin I and II Complexes License Full Estrogen Receptor $\alpha$-Dependent Enhancer Activation. Mol Cell 59, 188-202.

Lieberman-Aiden, E., van Berkum, N.L., Williams, L., Imakaev, M., Ragoczy, T., Telling, A., Amit, I., Lajoie, B.R., Sabo, P.J., Dorschner, M.O., et al. (2009). Comprehensive mapping of long-range interactions reveals folding principles of the human genome. Science 326, 289293.

Love, M.I., Huber, W., and Anders, S. (2014). Moderated estimation of fold change and dispersion for RNA-seq data with DESeq2. Genome Biol. 15, 550.

Magami, Y., Azuma, T., Inokuchi, H., Kokuno, S., Moriyasu, F., Kawai, K., and Hattori, T. (2002). Cell proliferation and renewal of normal hepatocytes and bile duct cells in adult mouse liver. Liver 22, 419-425.

Merkenschlager, M., and Nora, E.P. (2016). CTCF and Cohesin in Genome Folding and Transcriptional Gene Regulation. Annu Rev Genomics Hum Genet 17, 17-43.

Nagano, T., Lubling, Y., Varnai, C., Dudley, C., Leung, W., Baran, Y., Mendelson Cohen, N., Wingett, S., Fraser, P., and Tanay, A. (2017). Cell-cycle dynamics of chromosomal organization at single-cell resolution. Nature 547, 61-67.

Naumova, N., Imakaev, M., Fudenberg, G., Zhan, Y., Lajoie, B.R., Mirny, L.A., and Dekker, J. (2013). Organization of the Mitotic Chromosome. Science.

Nishide, K., and Hirano, T. (2014). Overlapping and non-overlapping functions of condensins I and II in neural stem cell divisions. PLoS Genet 10, e1004847.

Nora, E.P., Goloborodko, A., Valton, A.-L., Gibcus, J.H., Uebersohn, A., Abdennur, N., Dekker, J., Mirny, L.A., and Bruneau, B.G. (2017). Targeted Degradation of CTCF Decouples Local Insulation of Chromosome Domains from Genomic Compartmentalization. Cell 169, 930944.e22.

Nora, E.P., Lajoie, B.R., Schulz, E.G., Giorgetti, L., Okamoto, I., Servant, N., Piolot, T., van Berkum, N.L., Meisig, J., Sedat, J., et al. (2012). Spatial partitioning of the regulatory landscape of the X-inactivation centre. Nature 485, 381-385.

Nuebler, J., Fudenberg, G., Imakaev, M., Abdennur, N., and Mirny, L.A. (2018). Chromatin organization by an interplay of loop extrusion and compartmental segregation. Proc Natl Acad Sci U S A 115, E6697-E6706. 
Ono, T., Fang, Y., Spector, D.L., and Hirano, T. (2004). Spatial and temporal regulation of Condensins I and II in mitotic chromosome assembly in human cells. Mol. Biol. Cell 15, 3296-3308.

Ono, T., Losada, A., Hirano, M., Myers, M.P., Neuwald, A.F., and Hirano, T. (2003). Differential contributions of condensin I and condensin II to mitotic chromosome architecture in vertebrate cells. Cell 115, 109-121.

Pott, S., and Lieb, J.D. (2014). What are super-enhancers? Nat Genet 47, 8-12.

Rana, V., and Bosco, G. (2017). Condensin Regulation of Genome Architecture. J Cell Physiol $232,1617-1625$.

Rao, S.S.P., Huang, S.-C., Glenn St Hilaire, B., Engreitz, J.M., Perez, E.M., Kieffer-Kwon, K.-R., Sanborn, A.L., Johnstone, S.E., Bascom, G.D., Bochkov, I.D., et al. (2017). Cohesin Loss Eliminates All Loop Domains. Cell 171, 305-320.e324.

Rao, S.S.P., Huntley, M.H., Durand, N.C., Stamenova, E.K., Bochkov, I.D., Robinson, J.T., Sanborn, A.L., Machol, I., Omer, A.D., Lander, E.S., et al. (2014). A 3D Map of the Human Genome at Kilobase Resolution Reveals Principles of Chromatin Looping. Cell.

Sanborn, A.L., Rao, S.S.P., Huang, S.-C., Durand, N.C., Huntley, M.H., Jewett, A.I., Bochkov, I.D., Chinnappan, D., Cutkosky, A., Li, J., et al. (2015). Chromatin extrusion explains key features of loop and domain formation in wild-type and engineered genomes. Proc Natl Acad Sci U S A 112, E6456-E6465.

Santos, A., Wernersson, R., and Jensen, L.J. (2015). Cyclebase 3.0: a multi-organism database on cell-cycle regulation and phenotypes. Nucleic Acids Res 43, D1140-D1144.

Schmitt, A.D., Hu, M., Jung, I., Xu, Z., Qiu, Y., Tan, C.L., Li, Y., Lin, S., Lin, Y., Barr, C.L., et al. (2016). A Compendium of Chromatin Contact Maps Reveals Spatially Active Regions in the Human Genome. Cell Reports 17, 2042-2059.

Schuster, A.T., Sarvepalli, K., Murphy, E.A., and Longworth, M.S. (2013). Condensin II subunit dCAP-D3 restricts retrotransposon mobilization in Drosophila somatic cells. PLoS Genet 9, e1003879.

Schwarzer, W., Abdennur, N., Goloborodko, A., Pekowska, A., Fudenberg, G., Loe-Mie, Y., Fonseca, N.A., Huber, W., H Haering, C., Mirny, L., et al. (2017). Two independent modes of chromatin organization revealed by cohesin removal. Nature 551, 51-56.

Skarnes, W.C., Rosen, B., West, A.P., Koutsourakis, M., Bushell, W., Iyer, V., Mujica, A.O., Thomas, M., Harrow, J., Cox, T., et al. (2011). A conditional knockout resource for the genome-wide study of mouse gene function. Nature 474, 337-342.

Storey, J.D. (2003). The positive false discovery rate: A Bayesian interpretation and the qvalue. Annals of Statistics 31, 2013-2035. 
Tannour-Louet, M., Porteu, A., Vaulont, S., Kahn, A., and Vasseur-Cognet, M. (2002). A tamoxifen-inducible chimeric Cre recombinase specifically effective in the fetal and adult mouse liver. Hepatology 35, 1072-1081.

Tognini, P., Murakami, M., Liu, Y., Eckel-Mahan, K.L., Newman, J.C., Verdin, E., Baldi, P., and Sassone-Corsi, P. (2017). Distinct Circadian Signatures in Liver and Gut Clocks Revealed by Ketogenic Diet. Cell Metabolism 26, 523-538.e525.

Van Bortle, K., Nichols, M.H., Li, L., Ong, C.-T., Takenaka, N., Qin, Z.S., and Corces, V.G. (2014). Insulator function and topological domain border strength scale with architectural protein occupancy. Genome Biol. 15, R82.

van Ruiten, M.S., and Rowland, B.D. (2018). SMC Complexes: Universal DNA Looping Machines with Distinct Regulators. Trends Genet 34, 477-487.

Wang, J., Symul, L., Yeung, J., Gobet, C., Sobel, J., Lück, S., Westermark, P.O., Molina, N., and Naef, F. (2018). Circadian clock-dependent and -independent posttranscriptional regulation underlies temporal mRNA accumulation in mouse liver. Proc Natl Acad Sci USA 115, E1916-E1925.

Woodward, J., Taylor, G.C., Soares, D.C., Boyle, S., Sie, D., Read, D., Chathoth, K., Vukovic, M., Tarrats, N., Jamieson, D., et al. (2016). Condensin II mutation causes T-cell lymphoma through tissue-specific genome instability. Genes Dev 30, 2173-2186.

Wutz, G., Varnai, C., Nagasaka, K., Cisneros, D.A., Stocsits, R.R., Tang, W., Schoenfelder, S., Jessberger, G., Muhar, M., Hossain, M.J., et al. (2017). Topologically associating domains and chromatin loops depend on cohesin and are regulated by CTCF, WAPL, and PDS5 proteins. Embo J 36, 3573-3599.

Yuen, K.C., and Gerton, J.L. (2018). Taking cohesin and condensin in context. PLoS Genet 14, e1007118.

Yuen, K.C., Slaughter, B.D., and Gerton, J.L. (2017). Condensin II is anchored by TFIIIC and H3K4me3 in the mammalian genome and supports the expression of active dense gene clusters. Sci Adv 3, e1700191.

Zhang, T., Paulson, J.R., Bakhrebah, M., Kim, J.H., Nowell, C., Kalitsis, P., and Hudson, D.F. (2016). Condensin I and II behaviour in interphase nuclei and cells undergoing premature chromosome condensation. Chromosome Res 24, 243-269.

Zhang, Y.-K.J., Yeager, R.L., and Klaassen, C.D. (2009). Circadian expression profiles of drugprocessing genes and transcription factors in mouse liver. Drug Metab. Dispos. 37, 106-115. 


\title{
Supplementary Figures
}

\author{
Figure S1 - Replicates
}

A
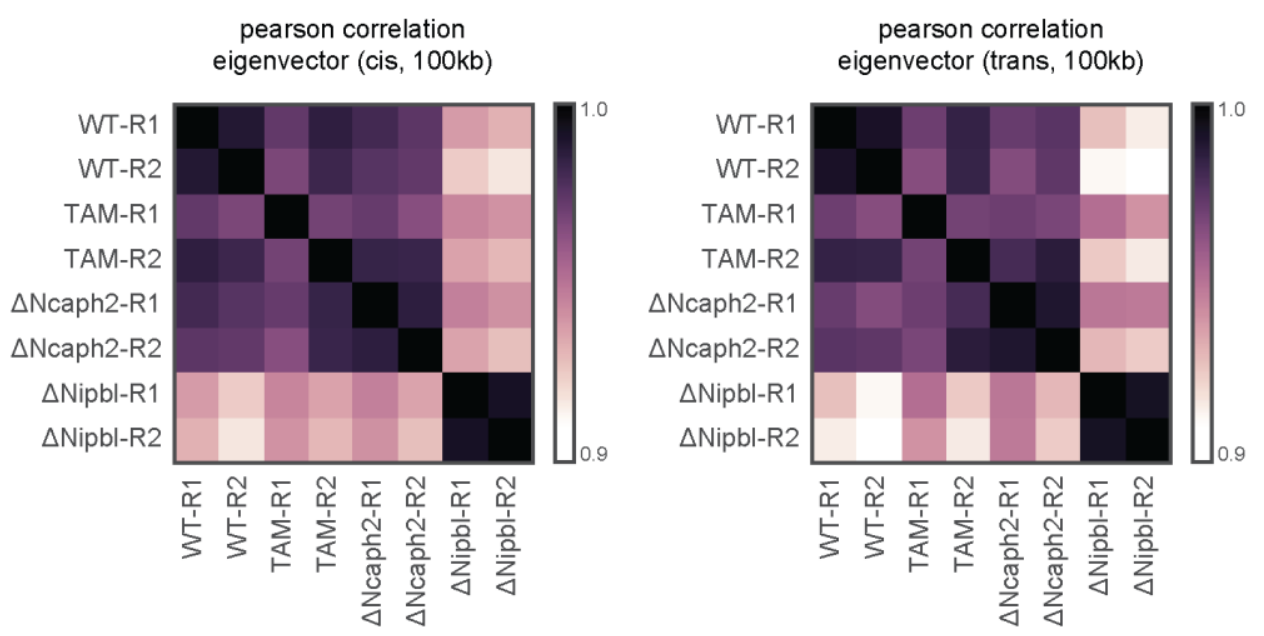

B
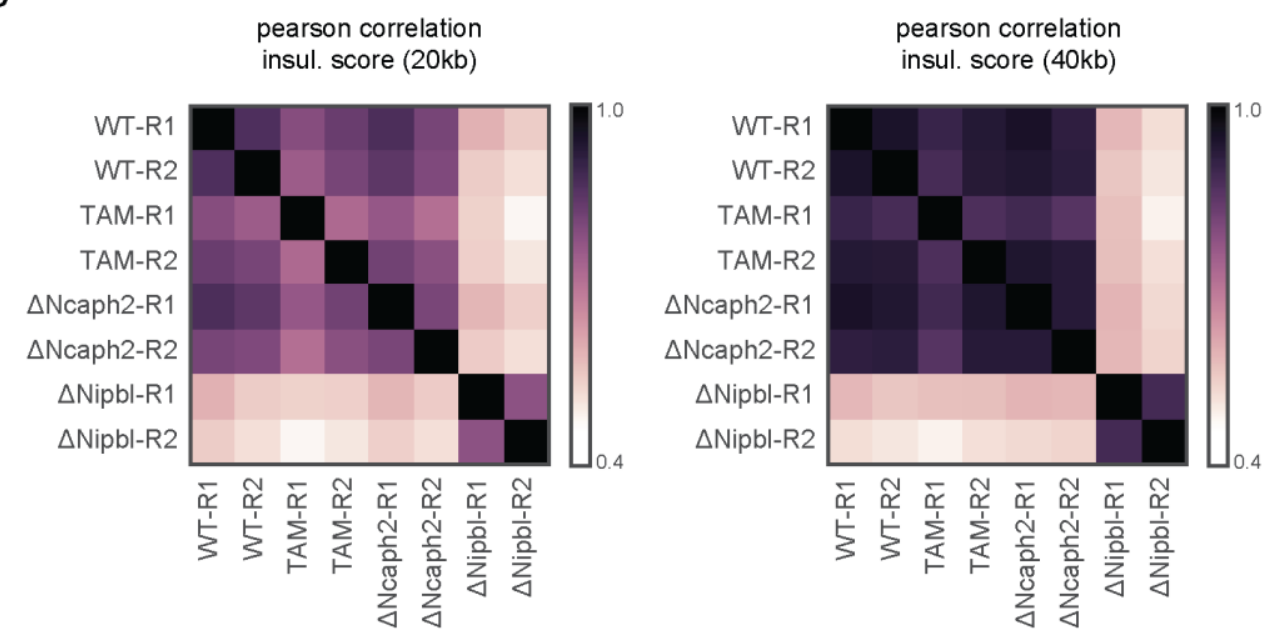

Pearson correlation scores comparing the different TTC experimental conditions and replicates for insulation score (A) eigenvector signal extracted from cis (left) and trans (right) at 100kb resolution and $(B)$ insulation score at $20 \mathrm{~kb}$ (left) and $40 \mathrm{~kb}$ (right) resolution. WT $=$ untreated 
bioRxiv preprint doi: https://doi.org/101101/437459; this version posted October 7, 2018. The copyright holder for this preprint (which was not certified by peer review) is the author/funder, who has granted bioRxiv a license to display the preprint in perpetuity. It is made available under aCC-BY 4.0 International license.

control (Nipb/ flox/flox $; \mathrm{Tg}(\mathrm{Ttr} \text {-cre/Esr1* })^{1 \mathrm{Vco}}$; no induction of Cre by tamoxifen injection). TAM = tamoxifen-injected control animals (Ncaph2 flox/flox ; TAM-injected; animals without $T g(T t r-$ cre/Esr1 $\left.\left.{ }^{*}\right)\right) . \Delta$ Ncaph2 $=$ Conditional Ncaph2 deletion $\left(N c a p h 2^{\text {flox/flox }} ; \operatorname{Tg}\left(T \operatorname{Tr} \text {-cre/Esr1 }{ }^{*}\right)^{1 V c o}-\right.$ TAM-injected). $\Delta \mathrm{Nipbl}=$ Conditional Nipbl deletion $\left(\right.$ Nipbfflox/flox $;$ Tg $\left(\text { Ttr-cre/Esr1 }{ }^{*}\right)^{1 \mathrm{Vco}}-\mathrm{TAM}$ injected) (Schwarzer et al., 2017). 


\section{Figure S2 - P(s) curves}

A

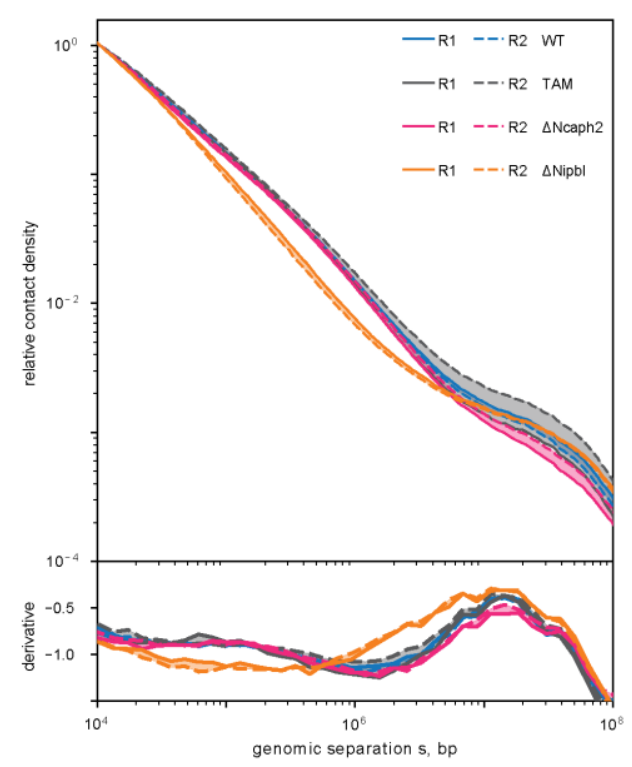

D
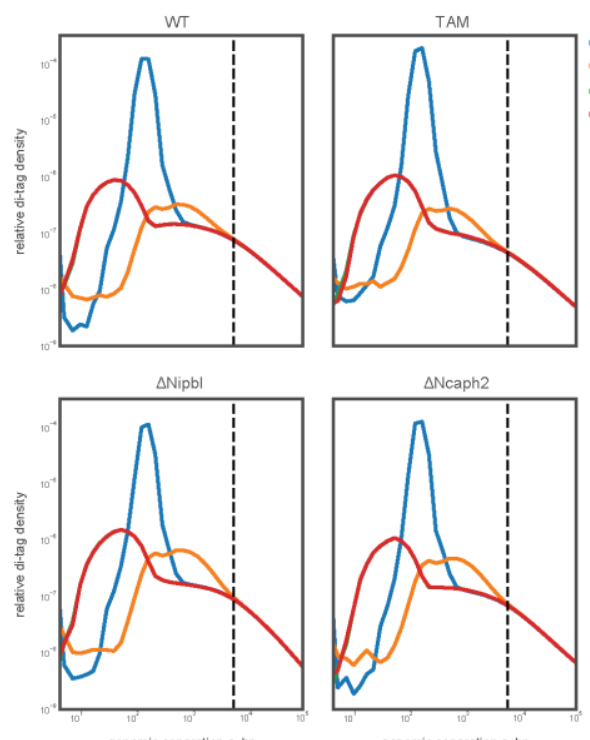

$\triangle \mathrm{Ncaph} 2$

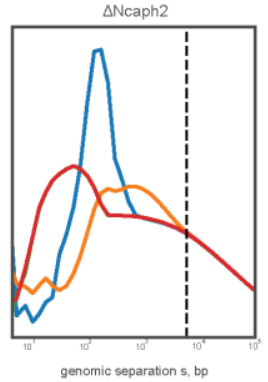

B

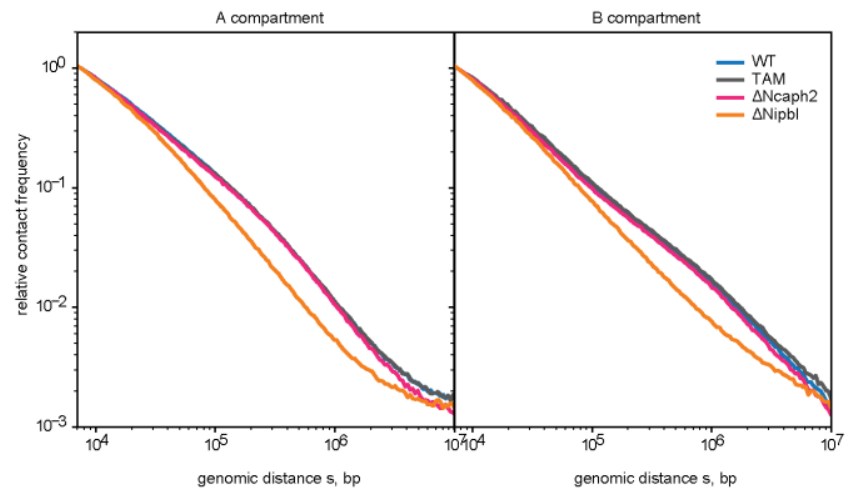

C

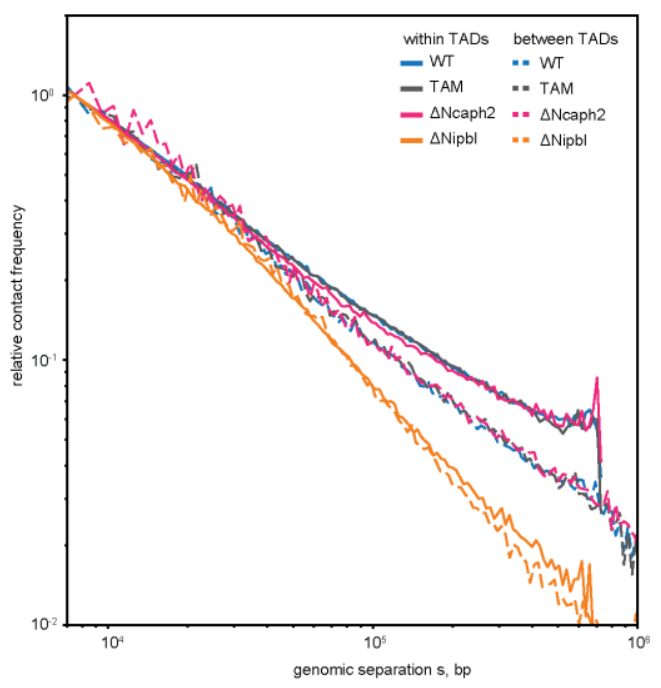

$P(s)$ curves by replicate and in sub-regions. (A) $P(s)$ curves as in Figure 2a but calculated separately for each replicate. The areas between curves from the same condition are shaded. (B) $P(s)$ curves for each condition, restricted to regions within A compartmental intervals (left) and $B$ compartmental intervals (right). The curves for $\triangle N C a p h 2$ and controls follow the same trend, and the shoulder in $B$ intervals is slightly more right-shifted (implying larger loop length in B regions). However, in both cases the $\triangle$ Nipbl shoulder is lost. (C) $P(s)$ curves within and between TADs called in WT. As observed in (Schwarzer et al., 2017), the shallower decay of the 
intra-TAD contact frequency scaling is lost in $\triangle \mathrm{Nipbl}$ and the two curves collapse. On the other hand, $\triangle$ NCaph2 curves match those of WT and TAM. (D) Short distance scaling curves of mapped read pairs split by strand orientations. The divergence of the curves reflects contaminant ligation products of the $\mathrm{Hi}-\mathrm{C}$ protocol. The point of convergence is used to determine a threshold for short distance filtering. 
Figure S3 - Insulation analysis

A

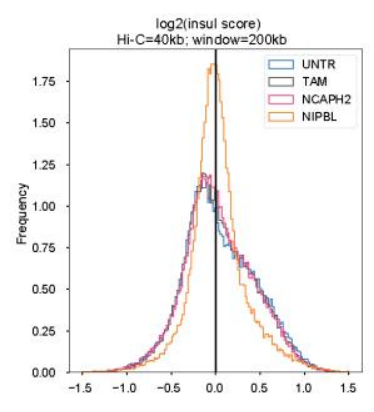

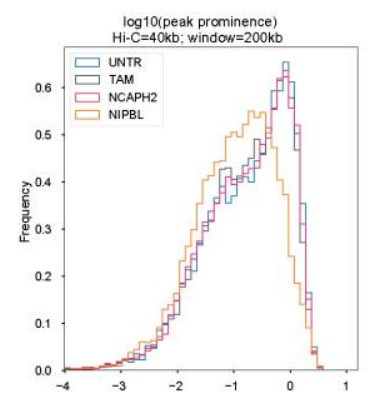

B

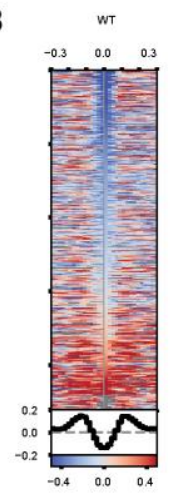

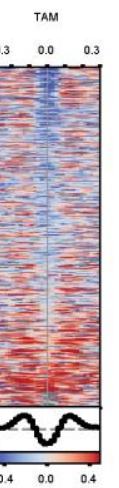
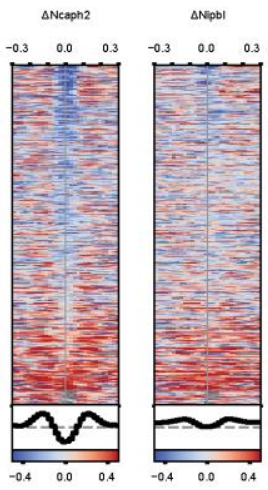

C
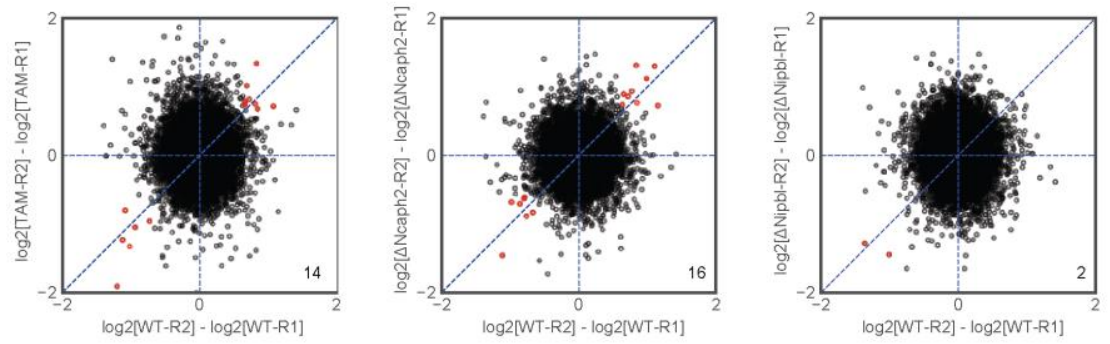

(A) Histograms of $\log _{2}$ insulation score (left) and $\log _{10}$ peak prominence (right). (B) Stacked heatmap of insulation scores at wildtype insulation valleys (as in figure2k). (C) As a control for differential insulation analysis, we compare intra-condition log fold changes of replicates using the same background variance estimate. The number of hits is printed in the bottom right corner. 


\section{Figure S4 - Aggregate paired landmarks (super-enhancers)}

A mouse liver "super-enhancers" (dbSuper)

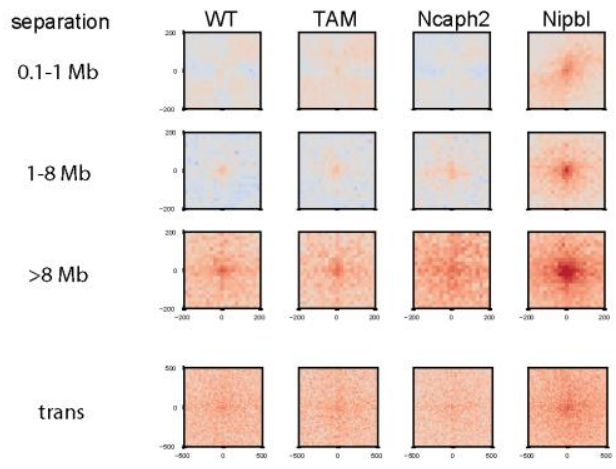

B H3K27ac clusters, length $\geq 10 \mathrm{~kb}$

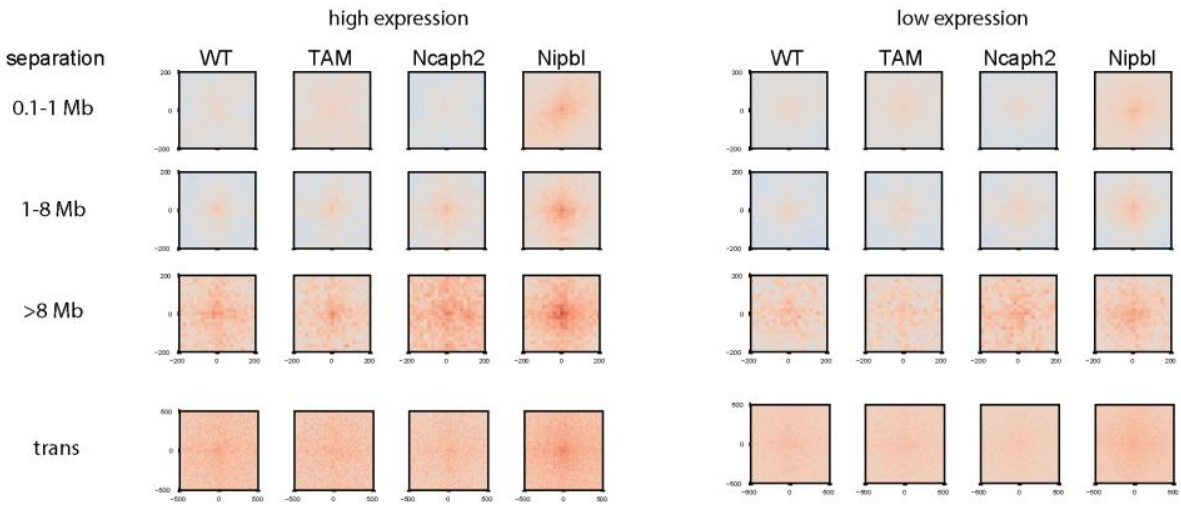

C H3K27ac clusters, length $<10 \mathrm{~kb}$

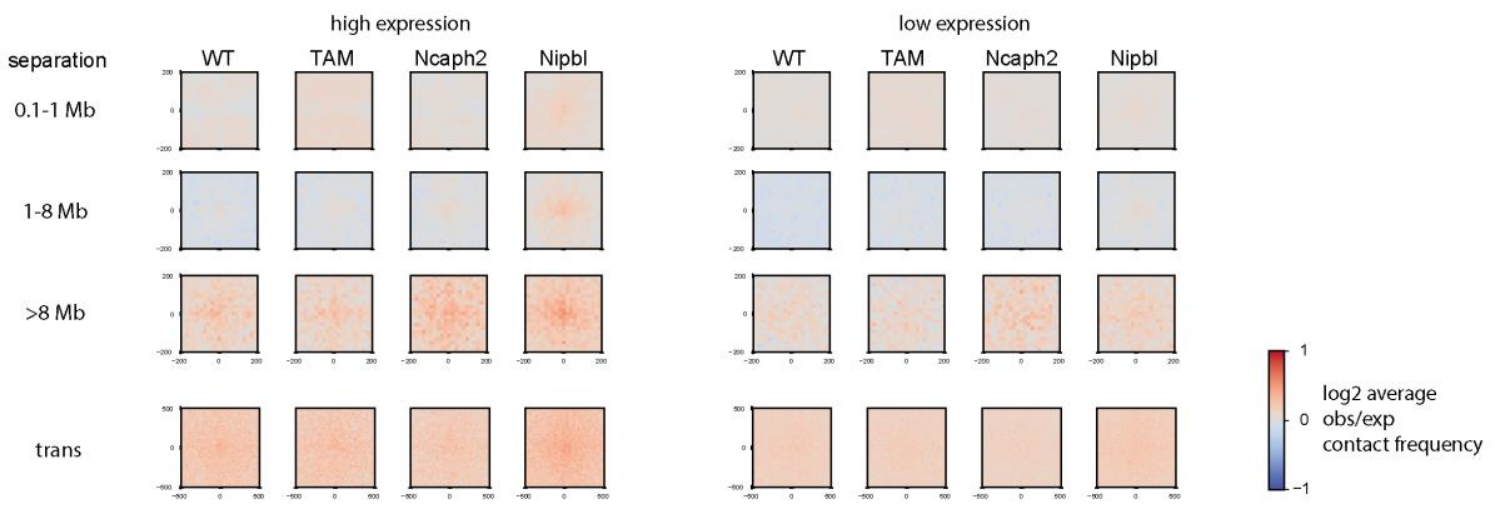

Aggregate $\mathrm{Hi}-\mathrm{C}$ pileup maps of pairs of super-enhancers stratified by genomic separation in cis and in trans. (B) Aggregate Hi-C pileup maps of pairs of long, stitched H3K27ac peaks not selected for super-enhancer status, but rather divided into two equal sized expression groups by the mean RNA-seq coverage measured in WT. (C) Same, but for the remaining unstitched H3K27ac peaks. 
Figure S5 - Aggregate paired landmarks (Pol3, TFIIIC)

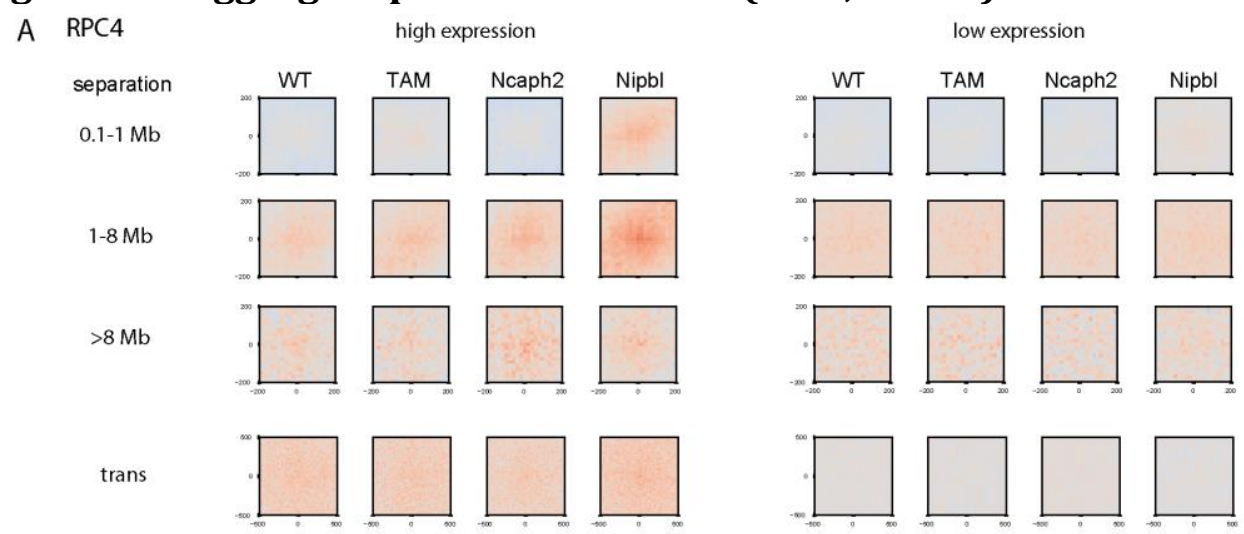

B RPC1

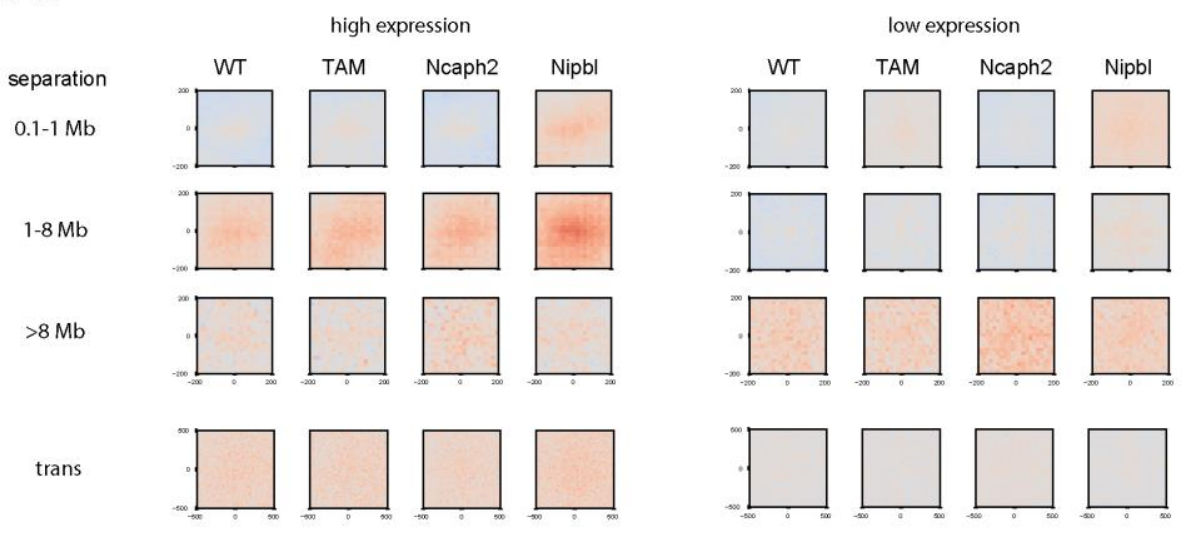

C TFIIIC

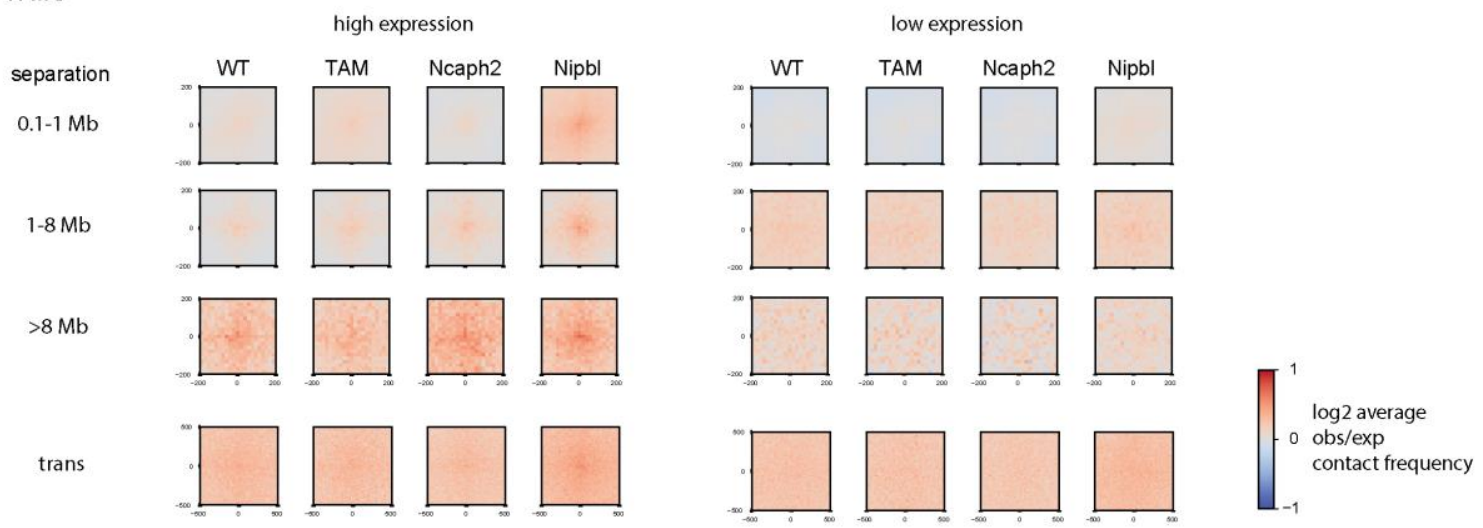

Aggregate Hi-C pileup maps of genomic landmarks stratified by genomic separation in cis and in trans: pairs of binding sites for RNAP-III associated factors (A) RPC4/POLR3D and (B) RPC1/POLR3A from mouse liver (Canella et al., 2012); (C) pairs of binding sites for TFIIIC measured in mouse ES cells (Yuen et al., 2017). Loci were divided into two equal sized expression groups by the RNA-seq coverage measured in WT. 


\section{Figure S6}
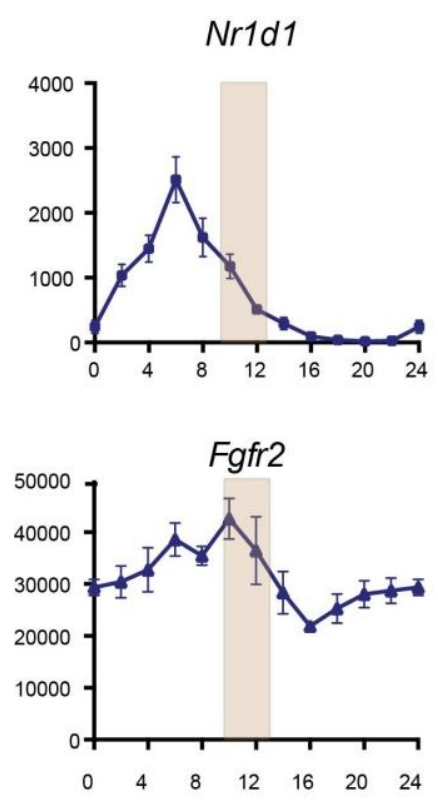

Rnf152

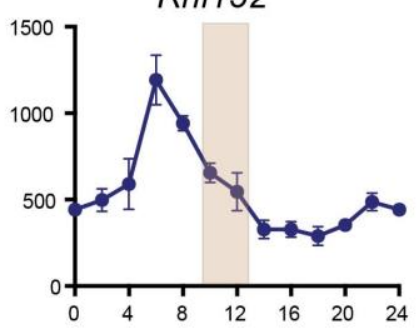

Cdkn1a
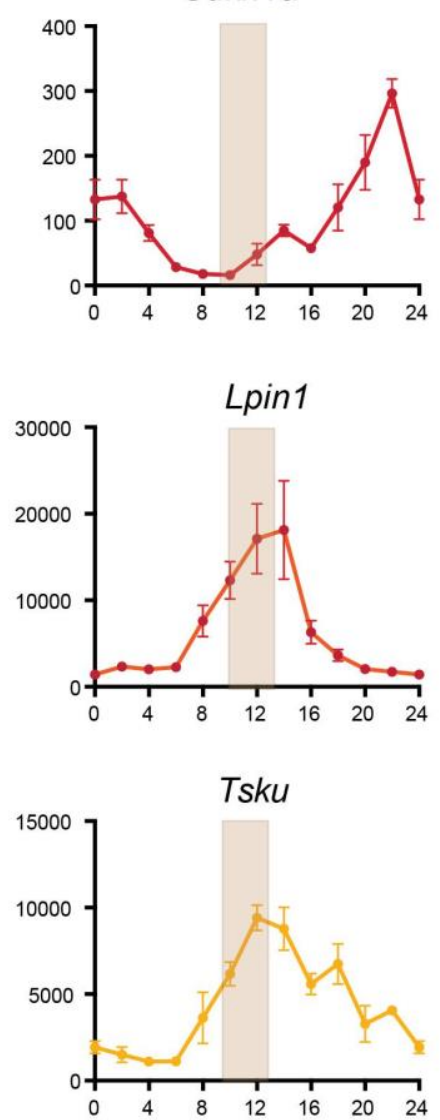

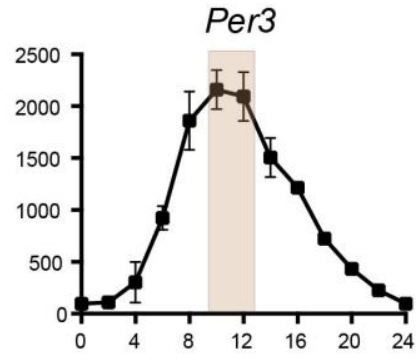

Circadian expression of several genes with expression differences between $\Delta$ Ncaph2 and TAM controls. Relative expression levels are plotted over a $24 \mathrm{~h}$-period. In blue (resp. red/orange), genes which are downregulated (resp. up-regulated) in $\Delta$ Ncaph2 samples. Down-regulated genes correspond to early-peak $(\mathrm{ZT}=6-8)$, while up-regulated ones peak latter in the day $(\mathrm{ZT}>15)$ (see Table 1). The brown shaded bar show the period during which samples have been collected. Circadian genes (e.g. Per3) which expression is stable during that period do not show expression changes between $\Delta$ Ncaph2 and controls. Data from (Fang et al., 2014; Wang et al., 2018). 
bioRxiv preprint doi: https://doi.org/10.1101/437459; this version posted October 7, 2018. The copyright holder for this preprint (which was not certified by peer review) is the author/funder, who has granted bioRxiv a license to display the preprint in perpetuity. It is made available under aCC-BY 4.0 International license.

Table S1 - Hi-C mapping statistics

\begin{tabular}{|l|l|l|l|l|l|l|}
\hline sample & codename & raw read pairs & valid pairs & cis & trans & cis:total \\
\hline WT-R1 & 014637Untr & $139,343,707$ & $53,326,649$ & $41,809,285$ & $11,517,364$ & 0.78 \\
\hline WT-R2 & 019440Untr & $114,258,179$ & $42,577,162$ & $33,363,853$ & $9,213,309$ & 0.78 \\
\hline WT & & & $98,775,892$ & $75,173,138$ & $23,602,754$ & 0.76 \\
\hline TAM-R1 & 014199 TAMcontr & $94,032,309$ & $14,065,226$ & $12,075,375$ & $1,989,851$ & 0.86 \\
\hline TAM-R2 & 014399TAMcontr & $134,045,253$ & $51,685,051$ & $39,445,882$ & $12,239,169$ & 0.76 \\
\hline TAM & & & $67,529,373$ & $51,521,257$ & $16,008,116$ & 0.76 \\
\hline$\Delta$ Nipbl-R1 & $014200 N i p b l$ & $131,409,162$ & $43,074,909$ & $32,545,604$ & $10,529,305$ & 0.76 \\
\hline$\Delta$ Nipbl-R2 & $016350 N i p b l$ & $133,914,046$ & $50,686,600$ & $38,098,292$ & $12,588,308$ & 0.75 \\
\hline$\Delta$ Nipbl & & & $95,471,646$ & $70,643,896$ & $24,827,750$ & 0.74 \\
\hline$\Delta$ Ncaph2-R1 & $014398 N c a p h 2$ & $127,595,875$ & $42,011,634$ & $31,569,049$ & $10,442,585$ & 0.75 \\
\hline$\Delta$ Ncaph2-R2 & $014953 N c a p h 2$ & $116,070,234$ & $42,168,415$ & $29,788,754$ & $12,379,661$ & 0.71 \\
\hline$\Delta$ Ncaph2 & & & $86,316,825$ & $61,357,803$ & $24,959,022$ & 0.71 \\
\hline
\end{tabular}

